\title{
A systematic review of voxelization method in additive manufacturing
}

\author{
Antonio Bacciaglia*, Alessandro Ceruti, and Alfredo Liverani \\ Department of Industrial Engineering (DIN), University of Bologna, Viale del Risorgimento 2, Bologna, Italy
}

Received: 1 June 2019 / Accepted: 21 June 2019

\begin{abstract}
Additive manufacturing (AM) is becoming an important alternative to traditional processes. AM technology shows several advantages in literature, and its use increases in aerospace, automotive and biomedicine. Time reduction in design-to-manufacturing cycle, customization, capability to generate complex shapes in one piece and ability to imitate low-weight bio-inspired shapes are the strength of designs based on AM. Due to its potentials, major progresses were done in AM, thanks to technology evolution and increased computational power. With regard to AM, voxelization can be defined as part's discretization in hexahedral elements, as done with pixels in 2D image. Voxels are used to speed-up geometry and algebraic manipulation thanks to their inherent advantages. This paper analyses advantages and criticalities of AM and voxel manipulation through a systematic literature review methodology. The analyses are based upon the filtering of a huge amount of publications available in literature up to obtaining the most significant 25 studies published in the last 5 years. The study's main result is the technology gap's identification, i.e. where AM and voxelization still need improvements, thus providing the reader with suggestions about possible further studies. Computer elaboration power and voxel discretization algorithms are suggested being key issues in AM's further development.
\end{abstract}

Keywords: Additive manufacturing / Voxel / Design / Systematic literature review (SLR)

\section{Introduction}

Additive manufacturing (AM) is becoming a widely used term in the engineering field. It describes a new way to manufacture components based on the idea of adding material layer by layer, as opposite to the traditional manufacturing processes based on chip removal or casting, milling, lathing processes where several design constraints must be respected [1]. AM is also known to the large audience with the popular name of $3 \mathrm{D}$ printing or rapid prototyping $(\mathrm{RP})$. These latter two terms are a bit restrictive and improper, because they do not describe all the AM potentiality: nowadays, this kind of technology is used not only to produce aesthetic or functional prototypes, but also parts to be installed in products available to endusers. In the last few years, industrial engineering has seen a significant growth of AM technology application in the manufacturing scenario, due to the advantages shown in cases where AM has been selected. The capability to generate complex shapes in one piece is advantageous to spare the time wasted in setting properly bolting connections or parts welding. Moreover, one-piece parts are more reliable

\footnotetext{
*e-mail: antonio.bacciaglia2@unibo.it
}

respect to an assembly of bolted parts (e.g. connections in vibrating environment where time has to be spent in maintenance to check connections) or welding (non destructive tests are not necessary to prove the quality of the welding spots). Significant advantages in terms of strength (or stiffness) to weight ratio can be obtained through AM because of the high freedom of shaping given to the designer, concept well captured by the expression: "What You See Is What You Build" [1]. This sentence stresses the attention on the fact that with traditional machining the shape must comply with constraints given by the manufacturing technology (e.g. no undercut in casting), while with AM there are few by far limits in shapes. Topology optimization (TO) algorithms and software packages conceived to perform structural optimization can suggest to the designer bio-inspired shapes with an un-presented structural efficiency.

There are several technologies which can be used to obtain AM parts: Selective Laser Sintering (SLS), Direct Metal Laser Sintering (DMLS), Powder Bed Fusion (PBS) can be applied to obtain high strength solid parts starting from metallic powders of iron, steel, titanium and aluminium. Stereolitography (SLA) allows the shaping of parts with low roughness and small details. In this not 
inclusive list it is worth motioning the Fused Deposition Modelling (FDM) technique which is wide spread among $3 \mathrm{D}$ printing practitioner due its low cost both in printer acquisition costs and in row material: a thin plastic wire (PLA or ABS) is melted in a hot head and added layer by layer to the part being printed. About AM typical uses, they range from parts to be installed in commercial aircraft to components designed by Formula 1 racing teams, up to technology practitioners developing Do-It-Yourself (DIY) projects [2] with FDM.

All the users, designers, and engineers working in the AM field are familiar with the word "voxelization". This is a recurrent keyword that describes the discretization of an object prior to its manufacturing to speed up geometrical and algebraic manipulation in CAD software [3]. In other words, the object is translated into a 3D matrix, where each cell represents a "voxel", the equivalent tri-dimensional unitary element of the pixel. Each voxel is initialized with a binary value based on the belonging of that element to the component or a local material density value [4] can be set to simulate multi-density bodies.

Voxelization allows to easily manage all the geometrical operations (boolean, slicing, rotation, etc.) that other software based on different methodology would do with some difficulties, with large models. On the other hand, voxelization is a geometry discretization which implies approximations in the shape representation: after voxelization, the external shape of a part is slightly changed, based on the dimensions of voxels. However, the voxelization advantages are magnified in case of complex bodies, like topologically optimized components or lattice structures, that can be manufactured only using AM. In this case the use of voxelization in TO can be useful to allow the reduction of time required to carry out the iterative analyses necessary to add or remove material in weak or excessively strong zones respectively.

Even though AM and voxelization are recurring keywords and many studies have been carried out in recent years, there are still some limitations and possible area of development to optimize the coupling between software to design AM and voxelization techniques. The aim of this work is to evaluate the state of the art of voxel-based representation to discuss the technology evolution and focus on advantages, limitations, and actual applications in the engineering field, in order to assess where we are and where to focus future researches. In order to achieve this goal, we carried out a rigorous literature review based on the Systematic Literature Review (SLR) methodology, which has been set thanks to the reproducibility and objectivity of the results [5] which are obtained in reviews where this methodology is applied.

This work is organized as follows: Section 2 describes step by step the application of the SLR methodology to voxel-related literature review. Section 3 contains the main results of the SLR, including a discussion of all the sources analysed to obtain the state of the art of AM and voxelization. Finally, Section 4 summarizes the results and suggests direction where to aim future researches in this field.

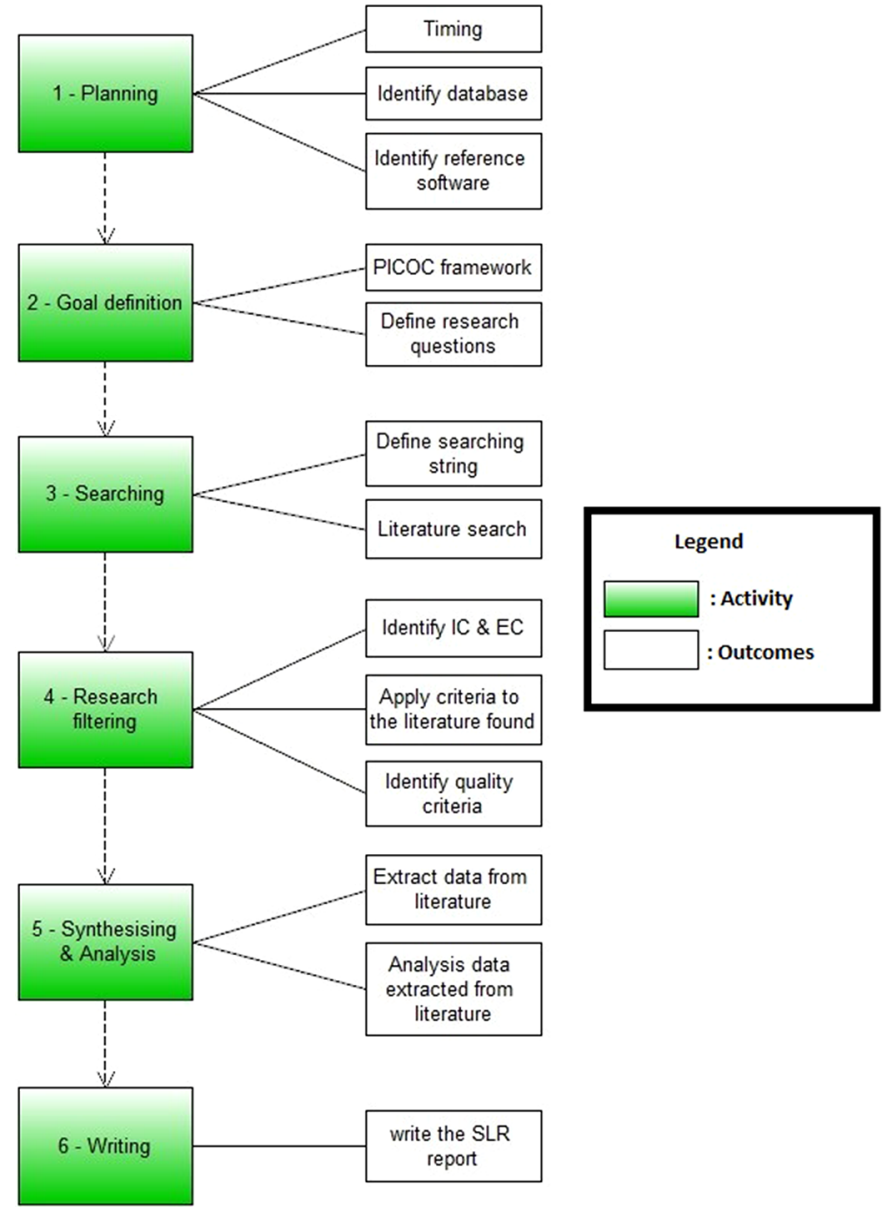

Fig. 1. SLR methodology scheme [5].

\section{Systematic literature review methodology}

To assess the state of the art of Additive Manufacturing with reference to voxelization methodologies, a systematic literature review (SLR) approach has been used. This strategy is composed of several steps, as described by Booth in the book "Systematic approaches to a successful literature review" [5]. This approach was applied in different works [6,7], in particular where authors required a rigorous analysis of the state of the art of selected topics. As previously said, this strategy is particularly suitable to capture literature gaps and possible areas of future development. The SLR methodology is based on several steps: planning, goal definition, searching, research filtering, synthesising and analysis and finally report writing, as depicted in (Fig. 1). In the following of this section, each step is described and its outcomes defined.

\subsection{Step 1 - Planning}

As the SLR methodology description suggests, this approach starts with a planning step. At this stage, it is important to identify the project timescale, the research database to be explored and the referencing system to be used. While dealing with AM and Voxelization, due to the rapid evolving of these technologies, authors agreed to 
take as a reference a timeline of few years, from 2013 to 2018. According to [6,7], we decided to use the following scientific databases to look for resources useful to satisfy the scope of this research:

- Scopus (www.scopus.com)

- IEEE Xplore (www.ieeexplore.com)

- Web of Science (www.webofknowledge.com)

- Engineering village (www.engineeringvillage.com)

In addition, Mendeley (www.mendeley.com) has been set the reference manager software because of its potentiality and user-friendly capabilities, in addition to JabRef (www.jabref.org), an open-source software capable to generate a .bib file needed for Latex text editor.

\subsection{Step 2 - Goal definition}

It is of straightforward importance to define in a clear way the research questions to answer in this study; this is necessary in order to clarify the task and define the scope of this literature review. To help the authors to identify them, a PICOC (Population, Intervention, Comparison, Outcomes and Context) framework is used to capture the key concepts of the state of the art [5].

For the present paper, the Population is identified with the additive manufacturing in industrial applications (automotive, aerospace, biomedical, civil, etc.). The Intervention consists of the utilization of voxel-based algorithms to draw or manipulate complex geometries that have to be manufactured. The Comparison can be carried out between the voxelization for geometrical discretization and for material deposition simulation. The Outcome can be identified in a key performance indicator, that is a parameter describing algorithms efficiency in terms of time needed to complete the task of voxelization. Finally, the Context includes industrial environment for the two $\mathrm{AM}$ and voxel-based algorithms items.

Thanks to the application of this framework, two research questions have been identified:

- Q1: What is the state of the art of voxel discretization algorithms to be used for typical complex shapes to be manufactured with Additive Manufacturing technologies?

- Q2: What are the potential future developments and possible new implementation of voxel-based algorithms in Additive Manufacturing?

\subsection{Step 3 - Searching}

Once defined the scope of the research, the SLR suggests to proceed browsing interesting publications and papers on the defined database (see Step 1) separately. This has been implemented by doing searches with the string "Additive manufacturing" AND "Voxel". As it can be noticed, the logical operator "AND" has been used in order to get only documents where both the topics are discussed. The database search resulted in 184 publications (whose number includes also duplicates) at the 10th of December 2018 date. It is worth noting that at this stage the SLR methodology does not include the reading of titles
Table 1. Detailed research outcomes for each database. The resulting number of publications and the fields, where the search string is applied, are reported in the table.

\begin{tabular}{lll}
\hline Database & Search field & N. of documents \\
\hline Scopus & Title - Abstract - & 74 \\
& Keywords & \\
Web of Science & Title - Topic & 47 \\
IEEE Xplore & Metadata Only & 4 \\
Engineering Village & Subject - Abstract - & 82 \\
& Title & 184 \\
\hline & Tot. & \\
\hline
\end{tabular}

or abstracts, but it requires a merely database search. Additional information regarding the outcomes have been collected in Table 1.

\subsection{Step 4 - Research filtering}

The following SLR methodology step requires the filtering and assessing of hundreds of documents resulting from Step 3. In order to choose the most relevant literature contributions, some inclusion and exclusion criteria are defined and applied iteratively to different documents. The inclusion criteria (IC) are:

- IC1: primary study that represents the use of voxelization for geometry manipulation and/or discretization in AM;

- IC2: primary study that represents the state of the art or application of AM and voxelization.

The exclusion criteria (EC) are:

- EC1: Not in English;

- EC2: Older than 2013;

- EC3: Not belonging to engineering or computer science field;

- EC4: Not applicable to Additive Manufacturing.

The IC and EC definition is based on previous studies [5-7] in order to decrease the huge amount of papers available in the selected database: in this way, only the newest and international contributions dealing with the voxelization application in industrial Additive Manufacturing are considered. These inclusion/exclusion criteria have been applied three times to each database in a consecutive way to all the documents emerging from Step 3. At the beginning, for the rough selection proper of the first stage, the IC and EC have been applied using the database filtering tools. Then, in a second stage, the remaining relevant documents have been analysed by checking the IC and EC compliance of the title and abstract; only at the last third stage, the IC and EC criteria have been applied to both introduction and conclusion, where usually authors summarize the contribution. The selection process is graphically shown in the flow chart included in (Fig. 2).

Thanks to the selection process above described, 25 out of 184 initial number of references have been identified relevant contributions. In order to detect the more 


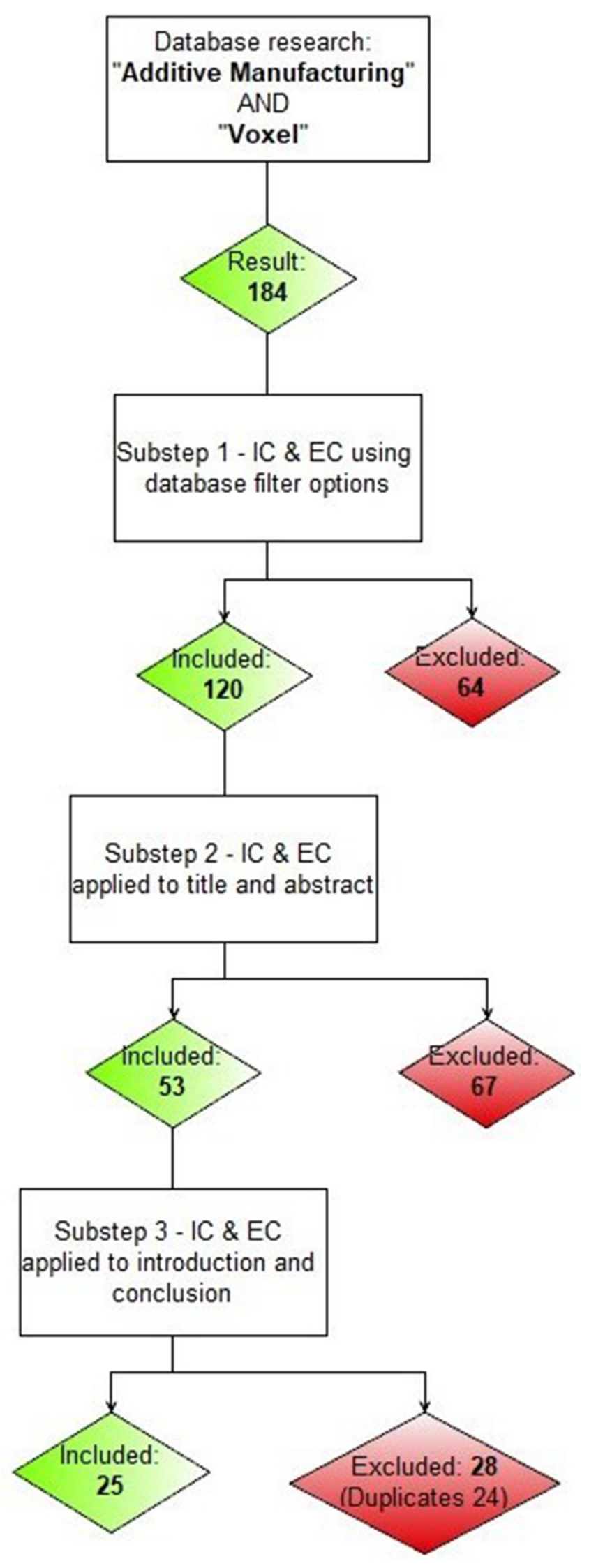

Fig. 2. Literature contribution selection using IC and EC applied at different stages.
Table 2. Quality criteria description.

\begin{tabular}{ll}
\hline & Description \\
\hline QC1 & Is the overall document clear? \\
QC2 & Is the methodology well exposed and clear? \\
QC3 & Is the contribution actual and not obsolete? \\
QC4 & Is the interaction between AM and voxel for \\
& complex shape manipulation present? \\
QC5 & Are the analytical results provided? \\
\hline
\end{tabular}

Table 3. Quality criteria application on the filtered publications. The last column includes the overall quality criteria scoring from zero to five.

\begin{tabular}{lllllll}
\hline Reference & QC1 & QC2 & QC3 & QC4 & QC5 & Tot. \\
\hline$[9]$ & 1 & 1 & 1 & 1 & 1 & 5 \\
{$[10]$} & 1 & 0.5 & 1 & 1 & 1 & 4.5 \\
{$[11]$} & 1 & 1 & 1 & 0.5 & 1 & 4.5 \\
{$[12]$} & 1 & 0.5 & 1 & 1 & 0.5 & 4 \\
{$[13]$} & 1 & 0.5 & 1 & 1 & 0.5 & 4 \\
{$[14]$} & 1 & 1 & 1 & 1 & 0 & 4 \\
{$[15]$} & 1 & 1 & 1 & 0 & 1 & 4 \\
{$[16]$} & 1 & 1 & 0.5 & 0.5 & 1 & 4 \\
{$[17]$} & 0.5 & 1 & 1 & 1 & 0.5 & 4 \\
{$[18]$} & 1 & 0.5 & 1 & 0 & 1 & 3.5 \\
{$[19]$} & 1 & 1 & 0.5 & 1 & 0 & 3.5 \\
{$[20]$} & 1 & 0.5 & 1 & 0.5 & 0.5 & 3.5 \\
{$[21]$} & 1 & 0.5 & 0.5 & 0.5 & 0.5 & 3 \\
{$[22]$} & 0.5 & 0.5 & 1 & 0.5 & 0.5 & 3 \\
{$[23]$} & 1 & 0.5 & 1 & 0.5 & 0 & 3 \\
{$[24]$} & 1 & 0.5 & 0.5 & 0.5 & 0.5 & 3 \\
{$[25]$} & 1 & 1 & 1 & 0 & 0 & 3 \\
{$[26]$} & 1 & 0.5 & 1 & 0.5 & 0 & 3 \\
{$[27]$} & 1 & 0.5 & 0.5 & 0.5 & 0 & 2.5 \\
{$[28]$} & 0.5 & 0.5 & 0.5 & 0.5 & 0.5 & 2.5 \\
{$[29]$} & 0.5 & 0.5 & 0.5 & 1 & 0 & 2.5 \\
{$[30]$} & 0.5 & 0.5 & 0.5 & 0 & 0.5 & 2 \\
{$[31]$} & 0.5 & 0.5 & 1 & 0 & 0 & 2 \\
{$[32]$} & 1 & 0.5 & 0 & 0 & 0.5 & 2 \\
{$[33]$} & 0.5 & 0 & 0.5 & 0 & 0.5 & 1.5 \\
\hline
\end{tabular}

clear and relevant documents, some quality criteria have been defined, based on the approach reported by [8], and applied to all the resulting 25 documents. These quality criteria (QC), which are answer to questions related to the quality and relevance of the papers, have been collected in Table 2.

In particular, each one of the five quality criteria (Q1, .., Q5) has been applied to each one of the 25 selected documents, and a subjective score of $0,0.5$ or 1 is given depending on the assessment of contribution quality by the authors, given with integrity and to the best of their knowledge. In more details, 0 is given when the quality criteria is not satisfied, 0.5 when it is partially complied and 1 if the quality criteria is completely fulfilled. The outcome of this assessment is included in Table 3 which reports all the score for each work, and in the last column the total mark is obtained summing up the partial scores.

Even if the maximum care in judgement has been taken, the use of quality criteria produces subjective 
Table 4. Table helpful for analysis and synthesis of the 25 articles. This is an extract of the whole table with first 4 references.

\begin{tabular}{|c|c|c|c|c|}
\hline & {$[9]$} & [10] & [11] & [12] \\
\hline Engineering field & $\begin{array}{l}\text { Industrial - } \\
\text { Biomedical }\end{array}$ & Industrial & Industrial & Industrial \\
\hline AM application & TO & $\begin{array}{l}\text { Process } \\
\text { simulation }\end{array}$ & $\begin{array}{l}\text { Process } \\
\text { simulation }\end{array}$ & TO \\
\hline AM technology & SLS - FDM & LENS & FDM & Material jetting \\
\hline Software platform & Not available & Not available & Matlab & Monolith \\
\hline Voxel employment & $\begin{array}{l}\text { Geometry } \\
\text { discretization unit }\end{array}$ & $\begin{array}{l}\text { Geometry } \\
\text { discretization unit }\end{array}$ & $\begin{array}{l}\text { Geometry } \\
\text { discretization unit }\end{array}$ & $\begin{array}{l}\text { Material } \\
\text { deposition unit }\end{array}$ \\
\hline
\end{tabular}

results. However, the aim of the paper scoring with quality criteria is not to exclude further works from the research; on the contrary, it has been used to provide information on the contribution quality in order to focus the attention on more relevant documents, even if all the 25 articles provide worth contribution to the $\mathrm{AM}$ and voxelization field.

\subsection{Step 5 - Synthesising and analysis}

The SLR methodology moves on with the analysis and synthesis of all the resulting documents in order to answer to the research questions. For this reason, in the following all the 25 papers that contribute at most to the scope of this work are cited. It is worth nothing that afterwords additional sources have been included to provide the reader with a better understanding of these contributions. As suggested by [34], a table that picks up the highlights of each paper is needed to catalogue all the 25 contributions in order to obtain a better literature review (see Table 4 as a reference for first 4 papers). This table lists in column the 25 references and in row five categories and recurring concepts that help the authors to get information, statistics and so on from each single paper. The five characteristics selected by the authors are: engineering field of application, Additive Manufacturing application, Additive Manufacturing technology, software platform and voxelization aim. These fields have been selected bearing in mind the scope of the work. As a matter of fact, authors in their contributions cite the additive technology and the software or programming language they used to develop their project. On the other hand, if there is no mention of these data, a "Not available" labelling is adopted. In the following, the five categories are briefly described, giving some statistics and percentage on the 25 resulting documents.

\subsubsection{Engineering field}

By the expression "engineering field", it is meant the applicability sector of the corresponding research. The recurring fields are:

- Industrial

- Biomedical

- Civil

- Design

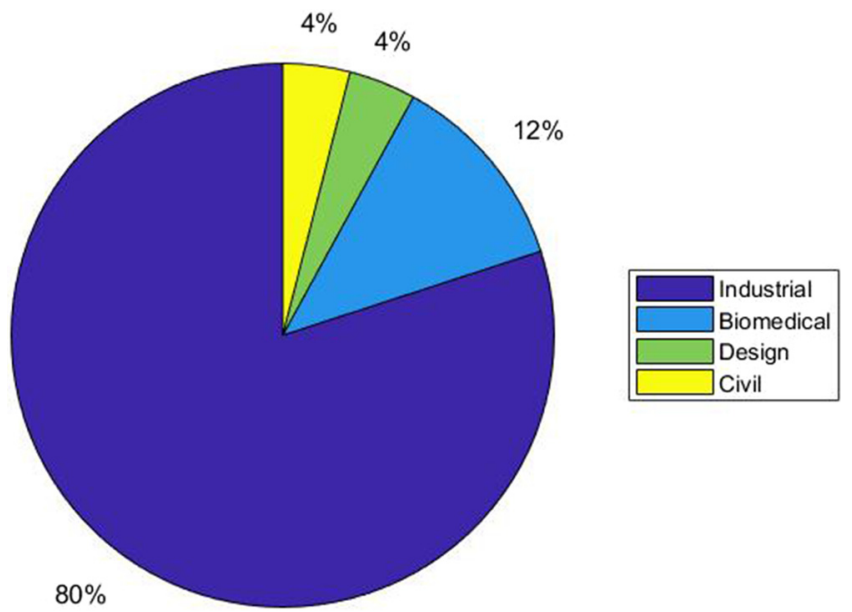

Fig. 3. Engineering field of the 25 contributions.

This subdivision comes from the different specifications for which each field is characterized, e.g. shape appealing for design, lightness for automotive and aerospace engineering, and so on. The data coming from this categorization are shown in Figure 3. There is a huge predominance of industrial field, while the second place is hold by the biomedical sector. This is in agreement with authors' expectations because industrial and biomedical are the current most significant fields for AM application.

\subsubsection{Additive manufacturing application}

With the label "Additive Manufacturing application" the authors mean the kind of task or technology that the Additive Manufacturing developed algorithms have to fulfil. These are identified by the following items:

- Process simulation

- Topology optimization (TO)

- Functionally graded material (FGM) generation

- Structure optimization

- Homogenization

- Additive manufacturing machine characterization

- Other

The data coming from this categorization are shown in Figure 4. The "other" category contains tasks such as lattice material degradation characterization, file format 


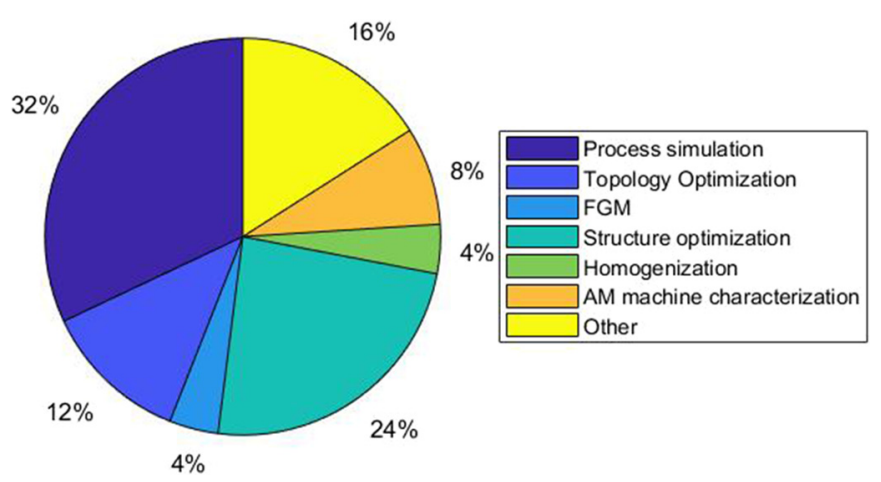

Fig. 4. Additive manufacturing application of the 25 contributions.

development for fiber-reinforced composites (FRC), smart material characterization and so on, that are not part of the most recurring items. Moreover, Topology Optimization and structure optimization seems very similar concepts: Topology Optimization [35] could be seen as a subset of the structure optimization techniques where the structure is optimized (i.e. weight reduction) simply by removing material from a domain set by the designer.

\subsubsection{Additive manufacturing technology}

With this expression, authors try to classify the different additive manufacturing processes employed in the 25 contributions, using the ASTM terminology [36]. Each adopted technique has peculiarities, advantages and limitations as discussed by [37], but the description of each technology is behind the scope of this work. This is the reason why this work doesn't include a deep analysis, but some interesting references for each technique are proposed to the reader.

- Fused deposition modelling (FDM) [38,39]

- Polyjet - Inkjet printing [40,41]

- Selective laser sintering (SLS) [42]

- Selective laser melting (SLM) [43]

- Laser engineering net shaping (LENS) [44]

- Stereolithography (SLA) [45]

- Fiber glass and resin [46]

- Lightweight concrete [47]

- Not available

The data coming from this categorization are shown in Figure 5. The reader can immediately notice a wide predominance of FDM technology due to its more affordable prices and its simplicity compared to other techniques like metallic powders based technologies costing more than millions of Euro and mainly available to companies.

\subsubsection{Software platform}

With the "Software platform" characteristic, this work aims to divide all the 25 references based on the programming language or software used to develop the algorithms of voxelization described in papers. In particular, the most recurring ones are:

- Matlab

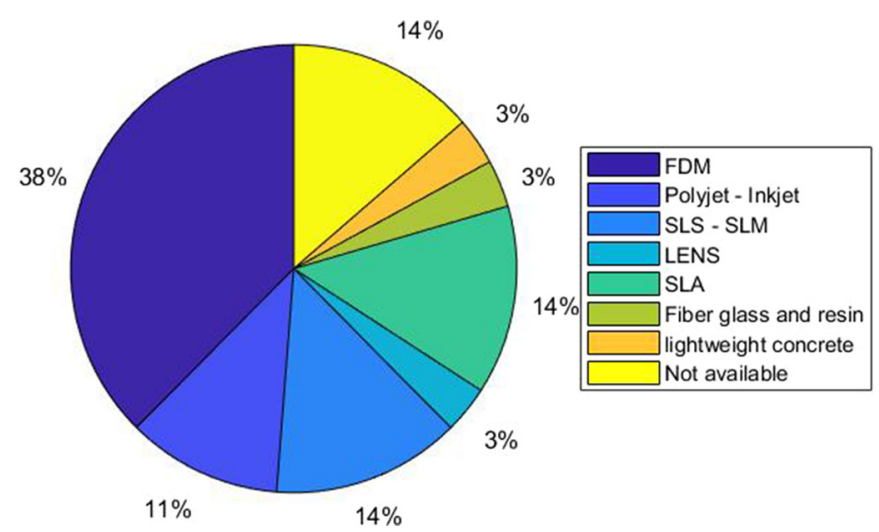

Fig. 5. Additive manufacturing technology of the 25 contributions.

- Grasshoper

- OpenCL

- Rhino

- Comsol...

Matlab and OpenCL (an open-source framework based on $\mathrm{C}$ ) give plenty of freedom in writing codes and scripts useful to implement and solve whatever mathematical model, and this is the reason behind the wide choice of such software platforms in the analysed contributions. Moreover, the Mathworks Company which trades Matlab offers a web space to community users where algorithms and codes can be shared.

\subsubsection{Voxelization usage}

The last categorization deals with the voxelization final aim. This geometry discretization, coupled with AM, could be exploited for different reasons:

- Geometry discretization unit

- Material deposition unit

- As matter constituent

- Image discretization unit

- Not available

As it could be seen in Figure 6, that collects all the data from the selected 25 papers, voxels are mainly used for geometry discretization to speed up geometry and algebraic manipulation and all the operations needed to view the component geometry prior to manufacturing it in AM. On the other hand, voxels are used as material deposition unit when inkjet technology is used (usually each drop of ink is represented by a voxel) or to simulate the material deposition in all the software packages where a framework to reproduce the AM process is developed.

\section{Results and discussion}

In this section a detailed analysis of each of the 25 selected contributions is included in order to answer the research questions Q1 and Q2. In particular, to answer the question Q1, that regards the state of the art discussion of voxel discretization for the handling of complex shapes 


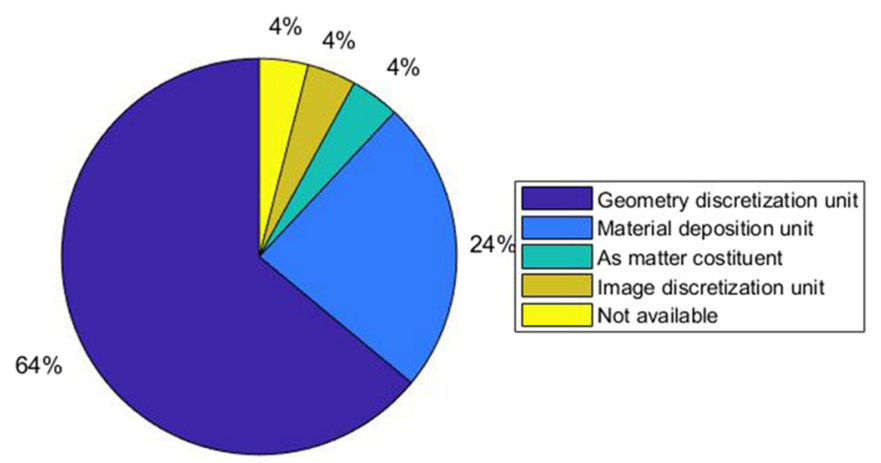

Fig. 6. Voxel-based discretization employment in the 25 contributions.

to be manufactured by AM technologies, the relevant papers will be divided according to the categories listed in Section 2, Step 5.

\subsection{Answer to Q1: What is the state of the art of voxel discretization algorithms to be used for typical complex shapes to be manufactured with Additive Manufacturing technologies?}

\subsubsection{Engineering field}

As Figure 3 shows, the majority of the 25 contributions refers to the industrial application, with a minor percentage of biomedical one, while only stand-alone examples refer to exterior design and civil applications. This trend is also confirmed by a recent AM survey [48] as well.

Regarding the exterior design, the paper [19] develops a discrete design method for large 3D AM structures in plastic material using robots to reduce "stair effect" problems. This effect is noticed when layers with finite thickness and different contour are stacked each other. The contribution proposes a discrete design fabrication method, serializing toolpath and solving errors with low computational cost, firstly at small scale (one voxel and its neighbours) and then at larger scale, avoiding global computations.

Moving to civil application, [27] designs a tool to design Functionally Graded Materials (FGM) for buildings using cement based aggregates for repairing applications. Thanks to a combination of Finite Element Analysis (FEA) and CAD voxelization, this tool is useful to select the best material to apply to a local region of a building, using the available material database to create an optimized structure. As expected, due to optimized material distribution, results always show an overall weight reduction along with material consumption minimization, while improvements in strength can be obtained as well.

When dealing with biomedical applications, it is well known that additive manufacturing is becoming very popular and various categories of biomedical materials are available [49]. The source [26] describes a new methodology to design simultaneously geometry and material for Graded Material components in AM applied in prosthetics, where high customization is needed. The methodology behind the project is based upon the determination of object's geometry and local material properties. The material is translated in a voxel-based manner for local composition as it is translated in 2D images with "halftoning" process. Then, these bitmaps are sent to a material jetting machine capable of multi-material generation. The prosthetic socket is generated in graded material and reinforced with composites. Reference [32] presents a methodology to reconstruct 2D Computed Tomography (CT) images heterogeneously and additively manufacture them. The boundary representation of common CAD software and STL file format are limited for inner part design in biomedical applications. To overcame this problem, this work proposes a topology-based methodology to represent and manufacture heterogeneous internal part of tissues by digitalizing CT images to get spatial porosity distribution function of the material. After topology reconstruction, an algorithm converts the topology information to process plan information: the inner porous structure is obtained using parallel cylindrical micro-filaments with a certain angle compared to the upper and lower layer. The porosity is defined as the void space remaining within the dense material and the filament spacing is obtained from the pixel intensity value previously acquired from CT image. All these data are saved and converted into a file format for bio-AM machine.

The paper [9] represents the last contribution that could be applied both in biomedical and in industrial field. This work presents a modified infill topology optimization algorithm inspired by bone-like porous structures, gathering advantages in terms of lightweight, resistance, strength and damage-tolerant properties that characterize these structures (Fig. 7). A 2D and 3D case study is shown and compared to the classic topology optimization results, showing great progress on the compliance reduction in case of external force variation or internal damage occurrence, satisfying biomedical requirements.

Moving towards industrial applications, where a relevant number of papers is available, the SLR selects $65 \%$ of references in the generic engineering field. Among the huge number of engineering research areas, automotive and aerospace industry relies on AM processes since high customization and lightness are mandatory. The source [10] develops an innovative voxel-based method for additive manufacturing process simulation useful to understand thermal behaviour of the deposition process. This is done in order to determine solidification rate, voids and residual stresses that affect the mechanical characteristics of a component using an optimized transient FEA. The main contribution is the developing of a mathematical model without the need to assemble the overall stiffness matrix and, as a consequence, speeding up the simulations. A similar theme is developed in [29], where another model to simulate the thermal processes for AM is proposed, by implementing an heat-conduction equations for isotropic mediums.

Dwelling in industrial applications, it can be noticed that AM is becoming an important alternative for functional part production: in such a scenario it is important to estimate the manufacturing costs, time and row material needed for AM parts production. The authors [21] formulate a framework for quotation of parts obtained through Selective Laser Synthesis (SLS) process, by estimating 

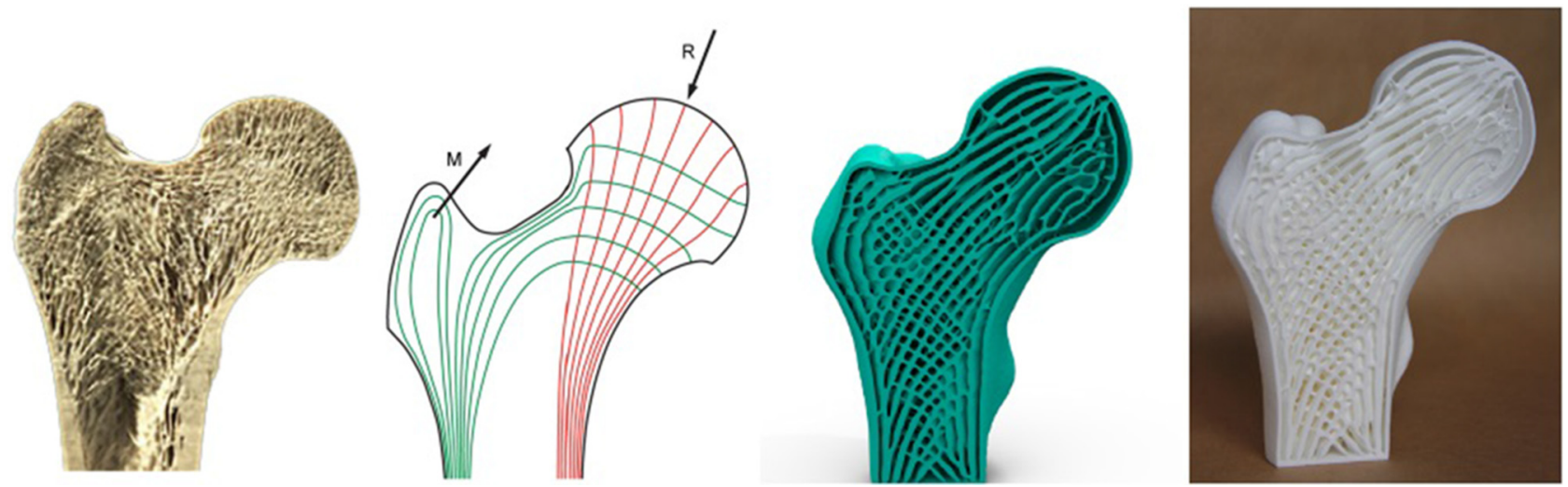

Fig. 7. Cross-section of an optimized bone [9].

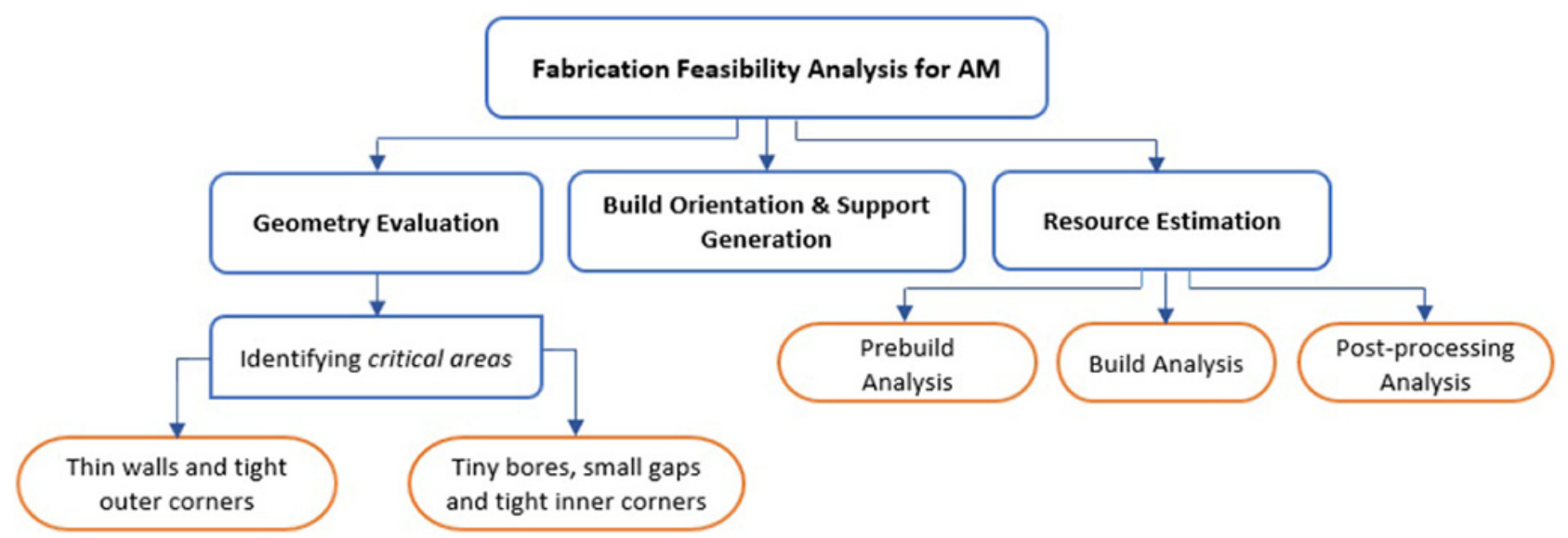

Fig. 8. AM framework for fabrication feasibility evaluation [11].

in real time material waste, energy consumption and building time. Moreover, the same framework is capable of optimizing the object orientation while minimizing the surface roughness and material waste. A similar approach is contained in $[11,20]$ where, in addition to the AM process quoting, authors developed an algorithm to investigate manufacturing feasibility in AM, including an optimized algorithm to create material support (Fig. 8).

The authors [28] design a virtual 3D manufacturing simulator in order to decrease printing and semantic errors. The algorithm prevents over- or under-extrusion and minimizes manufacturing errors. It is a real-time iterative process repeated for each layer that minimize computational costs by avoiding the overall component data storage. With respect to other simulators, a non-uniform filament deposition modelling is used, because of the nozzle acceleration and deceleration. Another process simulator is developed in [16] for 3D porous micro-structures and it is called VOLCO (VOLume COnserving model): it models the filament deposition in a virtual $3 \mathrm{D}$ voxel environment based on the simple assumption of the volume conservation. Even if several simplified models for material extrusion are available, all of them have limits in the prediction of complex 3D porous micro-structures with filaments widening. Moreover, [24] contributes to the topic with a framework whose aims is to measure the "distance" between the 3D model and the corresponding manufactured part. This is done introducing an hybrid approach that combines the Geometric Dimensioning and Tolerances (GD\&T) or Geometric Products Specifications (GPS) standards (based on B-rep visualization) and voxel-based modelling.

The work [12] presents an innovative workflow to create topologically optimized macro-components in the context of linear elastic theory, by optimizing the inner micro-structure to achieve a lightweight design: in such a way, material waste is minimized and material stiffness improves. This research focuses on the ability of design freedom, by limiting the micro-structure gamut to only one topology without bridging the micro and macro scales through homogenization approaches due to high computational costs. The recent published paper [18] describes a methodology based on topology optimization where manufacturing uncertainties are kept into account. This is done using a non-probabilistic strong method instead of a common deterministic approach [50].

Functionally Graded Materials FGM are used in industrial applications thanks to their gradual variation in composition and structure over the volume, thus obtaining variable mechanical properties in the material. The 


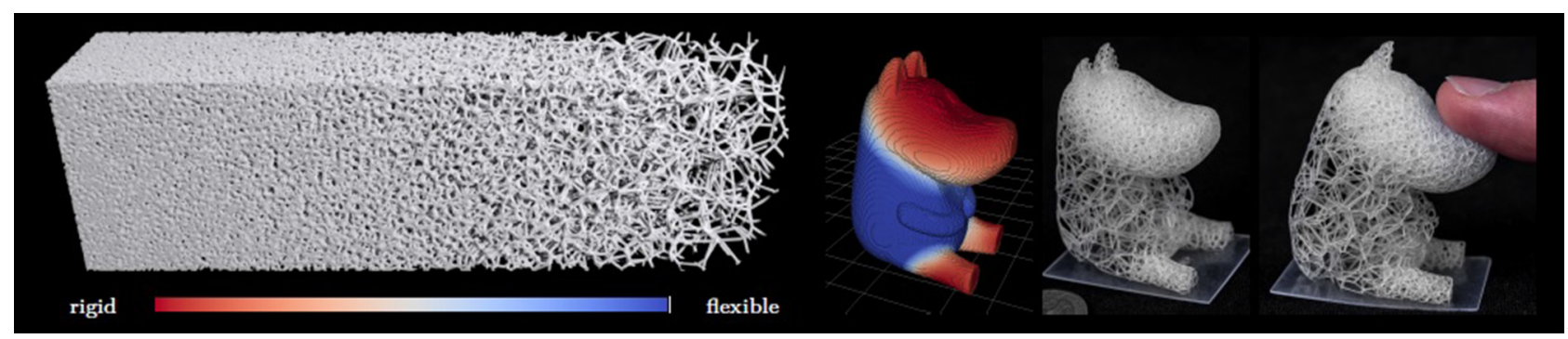

Fig. 9. Graded foam generation for AM [30].

paper [22] introduces a method to generate FGM that can be manufactured by AM techniques: it exploits the conversion from a gradient material to a part that could be printed using a limited number of materials available in a library.

Smart materials (SMs), which have one or more properties that can be significantly changed by external inputs such as stress or temperature, are used in applications such as aerospace and automotive. The source [23] develops a tool to design and simulate smart materials that can be manufactured thanks to AM. Four dimensional printing $(4 \mathrm{DP})$ is defined as the interaction between $3 \mathrm{D}$ printing technologies and SM thanks to the possibility of material optimization of one or more structural properties, like FGM or composites, even if SMs are still not diffused due to complex modelling of their response to an external input.

In industrial applications, as discussed several times, there is a strong need of optimized structures to reduce material waste, weight and so on. In this framework, [13] develops a tool to optimize material usage by hollowing the component in specific regions and optimizing the orientation using a weighted sum of AM performance parameters (build time, surface roughness and material utilization factor). Besides, multi-materials, like fiber reinforced composites, are nowadays very important because of the capability to produce structures with different materials in the interior boundaries. This kind of structures shows good potentials in fields where the lightweight design is crucial. [31] develops a new file format to describe fiber-reinforced composite parts to be produced by AM technologies.

The so called "lattice structures" are other complex kinds of materials, widely applied in the aerospace engineering: they are composed of repeated small elements, called cells, across a domain which generates a light and stiff component [51]. Cells are usually composed of trusses (e.g. 12 beams to form a cube) with a high void to dense material ratio.

As a consequence of the wide lattice structures external surfaces (usually cells are made by thin cylinders), stair effect could be problematic. Reference [14] measures the qualitative properties of tensile specimen made in lattice structure and investigate stair-step and deposition path effects, by changing building angle to get the numerical elastic modulus and ultimate tensile strength variation. Moreover, due to their high structure complexity, finite element analysis would require a huge amount of computational power. This is the reason why, homogenization algorithms have been developed by different research centres. One of the milestones in this context is the paper by Vigliotti [52] which contains the stiffness matrix model of some lattice unit cells. An extension of this work is contained in [15], where the authors consider both the semi-rigid joint effects and the effective structural parameters. Periodic lattice structures are used in industrial applications more than the stochastic dense materials, because of the easier way to extrapolate material properties [17]. On the other hand, a limited example of stochastic and aperiodic foams is present in literature; one of these examples is [30], where a procedure inspired by procedural texture is proposed: a texture is created using a mathematical algorithm rather than directly storing data. In this contribution, the resulting structure is found to be more simple to conform to a needed gradient, since it does not need special alignments and the behaviour is isotropic for large computed volumes (Fig. 9).

The main engineering fields of application have been extensively described. In general, as the reader can easily understand, each engineering field has different specifications and peculiarities that well reflect into AM potentials and characteristics.

\subsubsection{AM application}

The second characteristic for which the resulting 25 contributions have been analysed is the Additive Manufacturing application, or in other words, the kind of task or technology theme discussed.

According to Figure 4, almost one third of all the contributions is about process simulation. In particular, a recurring theme is the development of a framework for manufacturing quoting, that is basically an estimation of time, cost, or material consumption required to produce a component whose CAD model is available [11,20,21] while optimizing the part orientation and estimating its printing feasibility. On the other hand, $[10,29]$ focus the attention on the thermal simulation during the manufacturing process in order to assess its influence on the final product performances. This is done using a transient finite element analysis without requiring to assembly the overall stiffness matrix and it is solved with Newton-Raphson method. The paper [32] proposes a topology-based methodology to simulate the manufacturing process of heterogeneous internal part of tissues. The inner porous structure is 
a) $\mathbf{2 5 0} \mu \mathrm{m}$ layer thickness

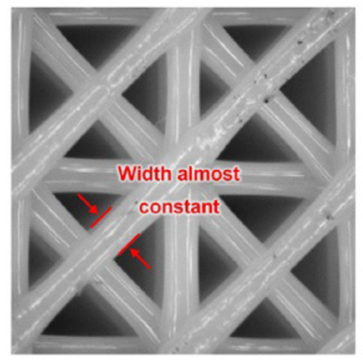

Experimental

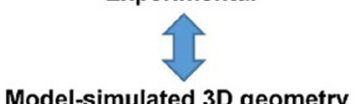

Model-simulated 3D geometry

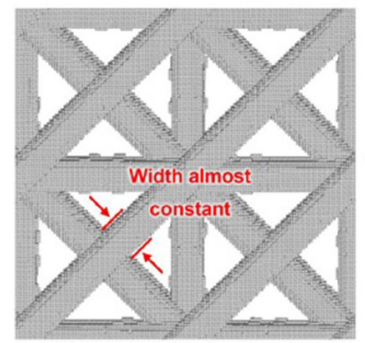

b) $100 \mu \mathrm{m}$ layer thickness

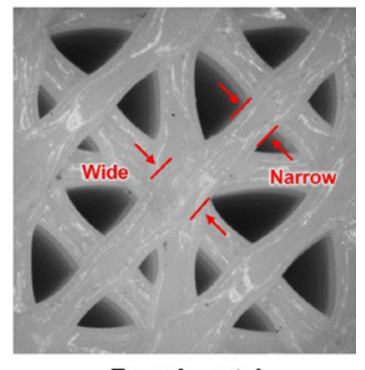

Experimental
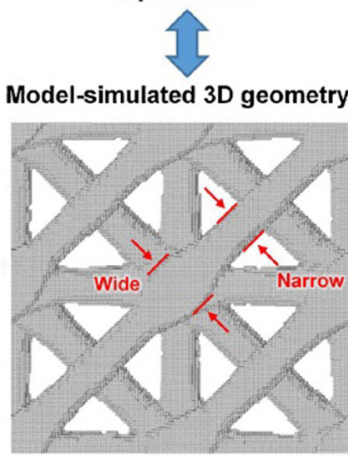

Model-simulated 3D geometry

Fig. 10. Virtual process simulation that takes into account the increase of filament width by volume conservation [16].

obtained using parallel cylindrical micro-filaments with a certain angle with respect to the upper and lower layer. The porosity is defined as the void space divided by the whole volume, and the filament spacing is obtained from the pixel intensity value previously acquired from $\mathrm{CT}$ images. [28] develops a framework to preview printing process whose aim is to find and correct syntax and semantic errors. All the printing information are contained in the G-code file which is the input of the simulator for virtual manufacturing, but also the basis for code debugging and fixing of possible errors. To increase the simulator fidelity, the author takes into account a mathematical model made of two steps: a deposition function to describe the deposition of a uniform filament and the diffusion process to get the filament deformations due to heat and cooling times. With reference to process simulation, [16] focuses on porous micro-structures. This paper tries to fill the gap in literature to simulate geometry variation due to fabrication process for porous structures. Even if several simplified model for material extrusion are available, they all have limits and no one can predict complex 3D porous micro-structures with multi-directional interaction between filaments. The same paper describes the filament deposition process in a virtual $3 \mathrm{D}$ voxel environment based on the simple assumption of volume conservation. Layer thickness (LT) is an important parameter that can change the structural mechanical properties of this kind of structures. The paper reports also experimental tests that have been carried out to study the effect of LT on porous fraction and of LT on compressive modulus through compressive test and FEM analysis, achieving good results in terms of filament width (Fig. 10).

Another important topic in AM is the topology optimization, which allows to create complex shapes knowing the maximum allowable volume, the applied forces and the constrains. An iterative process where a fitness function, that usually is the compliance [35] (internal stress times displacement of each voxel) is performed. This is an optimized way to design components when lightness and material strength have to be maximized as it happens in aerospace or automotive applications [53]. Taking into consideration the paper resulting from the SLR, [12] does not bridge the micro and macro scales through homogenization approaches due to the high computational costs required: on the other hand, this research focuses on the ability of increasing the design freedom, by limiting the micro-structure gamut to only one topology. The authors combined AM with TO, emphasizing the positive aspects of additively manufacturing processing, in a workflow divided in 3 steps: design automation process (where the classic TO problem is solved minimizing the compliance); material compilation process to obtain a physically realizable 3D object (including support material by a black and white 2D bitmap, where a tough and a strong material have to be placed); the fabrication process, thanks to an additive machine with high accuracy (which is capable of realizing complex shapes due to its resolution in the order of microns (Fig. 11).

An original approach is used in [18], where a nonprobabilistic strong method is implemented, in contrast with a common deterministic approach. The different approach reflects on higher computational cost, by increasing the number of unknown variables in order to reduce the manufacturing sensitivity, while increasing the results accuracy. A fixed grid in TO could output nonphysical stress concentration at jagger boundaries that affect the results, i.e. when the boundary of the structure is not aligned with the finite element mesh, in particular with voxel-based mesh. Authors present an example of a plate with a hole: increasing the hole radius, the resulting stress oscillates and overestimates the actual stresses. To solve this problem, an alternative two-step approach is proposed based on the introduction of a thin layer of intermediate material between voids, and a proper selection of the stress interpolation factor. The paper [9] describes an alternative to SIMP method (Solid Isotropic Material with Penalization [54]) focusing on the replacement of the global volume fraction constrain, usually implemented as in [50], with a local material accumulation in the proximity of a considered voxel, leaving the decision to set void or full voxel attribute to the optimizer. The mathematical model is rewritten properly to fit a numerical simulation and the discrete design variable (local density) is projected into a continuous one and smooths through a filtering operation to remove numerical instabilities. The projection becomes sharper (towards black and white solution) with increasing iteration, improving convergence behaviour.

The structure optimization, a general task in which TO is comprehended, is another recurring subject in literature. In this context, it is notable the study [13], where a tool is developed to optimize material usage, by hollowing the component. Due to this material reduction, external and internal supports are needed; this is done taking into account the material strength and the support 
1. Design Automation
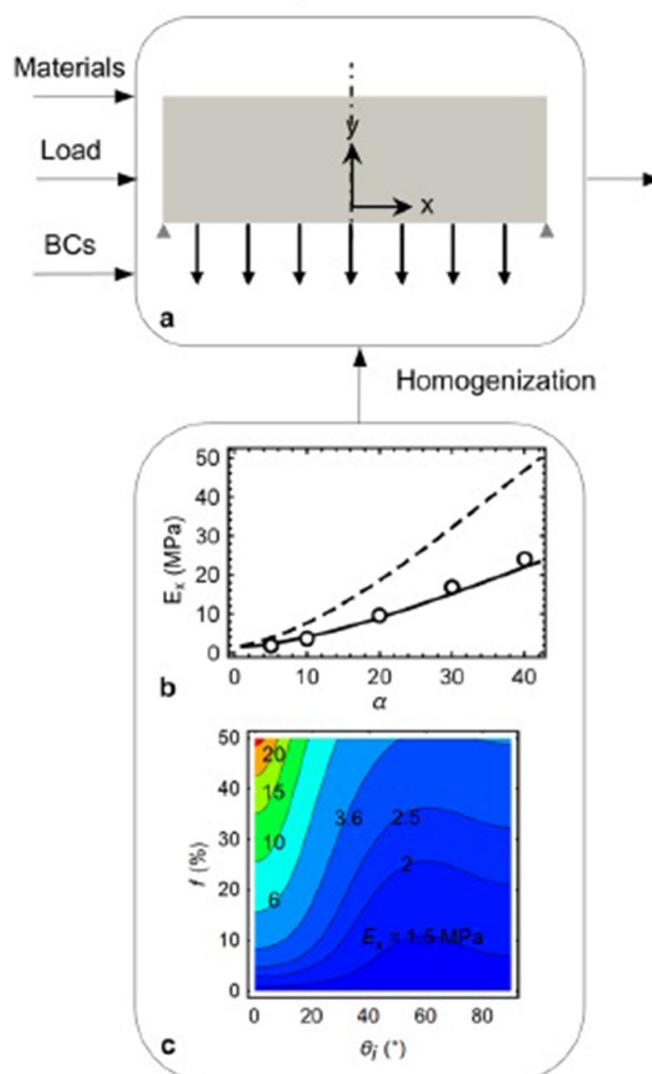

2. Material Compilation

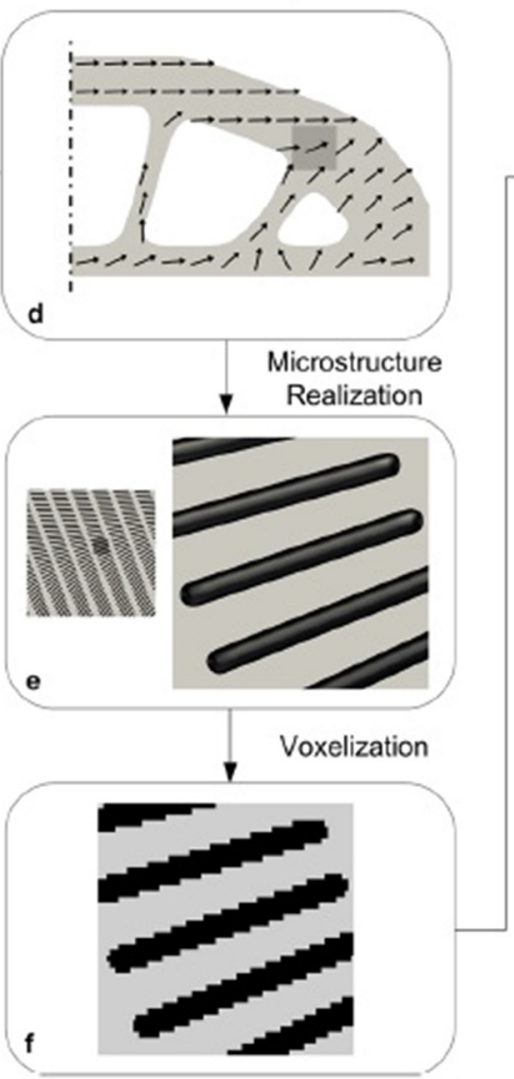

3. Digital Fabrication

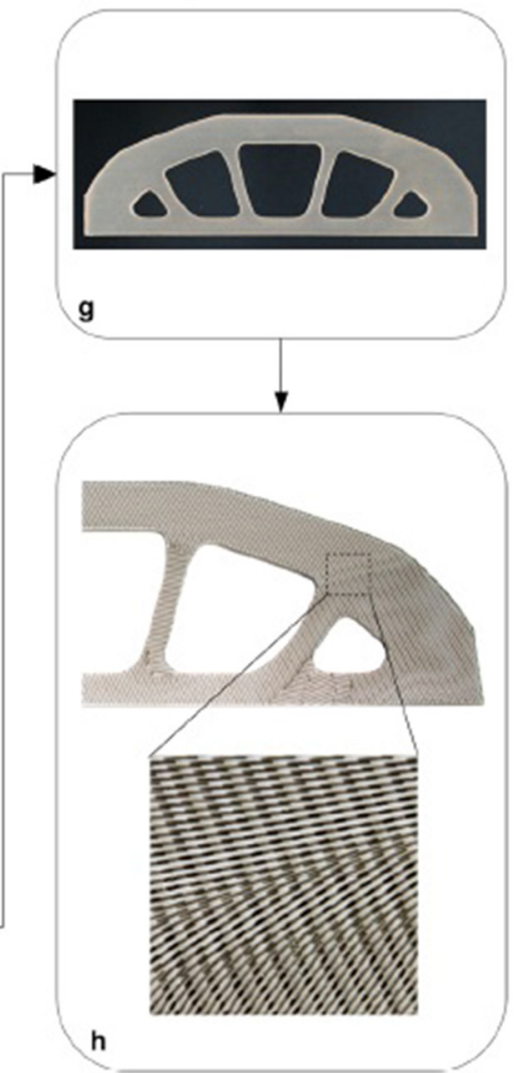

Fig. 11. Topology optimization methodology behind the contribution of [12].

is minimized and optimized thanks to a k-means clustering. Finally, an algorithm is used to find the optimal orientation based on the minimization of a fitness function. This is defined as the weighted sum of building time, surface roughness and material utilization factor. Another example dealing with similar concepts is [30] where an algorithm to generate Voronoi stochastic procedural micro-structures, or foams inspired by procedural texture is developed. The resulting structure is stochastic and aperiodic, which is more simple to conform to a needed gradient. These foams are described using local density and beam radius, assuming a smooth variation of density inside the foam to avoid discontinuities. For relative large foams, the distance between isotropic full material and procedural Voronoi foams is limited; the final outcome is that as the simulated volume increases, so the elastic tensor goes towards the ideal one. In the source [26], a tool to design simultaneously geometry and materials for graded material components is developed. It determines object's geometry and local material properties in the first step of running. Then the material is translated in voxel-based manner for local composition (bitmaps) as it is done in 2D images with half-toning process. The Bitmap file generation is made by 2 STL files, one for each material, written at voxel-scale level, to be able to smooth material transition. As a matter of fact, geometry bitmapping is a good strategy because files are written in binary to indicate where drops of each material should be jetted. Authors [19] develop a methodology to optimize large scale 3D AM machines. The design fabrication method is based on discreetness, serializing toolpath and solving errors with low computational cost, firstly at small scale (one voxel and its neighbours) and then at larger scale, aiming to local optimization, avoiding global computations. The discrete toolpath of local regions are then combined to obtain a continuous one, thus avoiding singularities and intersections using a combinatoric algorithm. In [27], the proposed algorithm, once FEM analysis results are known, is capable of deciding the position of a concrete material composition available on a predesigned library, in the regions of higher stresses compared to others, creating an optimized structure. Finally, [17] describes a methodology to design optimized lattice tessellated structures. This is carried out with a logical "AND" operation between the domain and the tessellated cell distribution, both represented in voxelized black and white (b/w) manner. The paper also focuses on adding a conformal skin, improving component integrity. The main idea behind the skin generation methodology is to construct a b/w voxel model of the skin. An algorithm evaluates whether the considered voxel belongs to the boundary or not and after that, the tessellated domain is eroded in its boundary in order to subtract the inner part, not useful for the net skin generation. The resulting domain is then projected by adding integral multiplies along the 3 axes by translating the voxel of a quantity selected to ensure that 


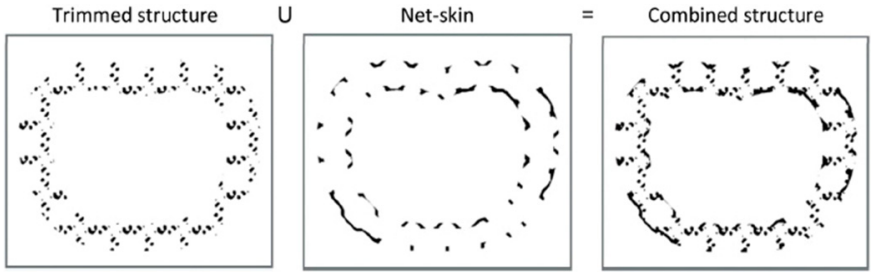

Fig. 12. Combination of trimmed lattice and net skin with logical AND operation [17].

all projections reach the boundary of solid skin (Fig. 12). This is a challenging operation especially for highly curvature surfaces. This project presents also the possibility to generate graded lattices by overlapping a grey scale image. Being available a lattice library a unit cell can be associated to different levels of grey.

Proceeding with the literature analysis, two contributions focus on the characterization of additive manufacturing machines. For example, [25] describes the characterization of micro additive process in terms of minimum voxel size with an iterative design of test parts to investigate resolution and repeatability. An iterative process, based on axiomatic design theory [55], is performed to identify and remove couplings, errors and flaws in the manufacturing process. The benchmark objects are studied using a microscope, because of the fact that micro-structures show thickness of some microns. On the other hand, [33] characterizes a macro-lattice assembly system to improve the reliability of structures, analysing the errors. The machine developed in this paper consists in an end-effector mounted in a 5-axis CNC machine. Each pre-manufactured lattice macro element is an octahedral structure made of glass fiber in thermoplastic resin. The end-effector is designed to be made of 2 components: a gripper assembly to held voxels in a specific position, and a bolter assembly fixes two voxels together and fastens them. The design goal of this element is to achieve high voxel placement accuracy and fine calibration.

The remaining contributions do not specifically belong to the previous categories, but they provide important information about the state of the art analysis. The work [22] develops a method to generate FGM by substituting the continuous material with 2 or more base materials available in a framework library in a discrete manner based on the gradient function. The FGM design is based on its matching with library patterns in grids with the tensor that describes the deformations under normal or shear stresses as a function of Young modulus which can variate inside the component, while keeping constant the Poisson ratio. An interesting topic for the industrial engineering applications is described in [23]: authors developed a tool to rapidly design and simulate smart materials that can be manufactured through AM technologies. Instead of a traditional FEA, a Mass-Spring System is used to rapidly model the component, with voxels used instead of points. The design process consists in 4 steps: geometry voxelization for easier handling, material distribution definition for each voxel, stimulus definition and material simulation.
In industrial applications where weight reduction is mandatory, such as automotive and aerospace, lattice structures are widely used [56]. Reference [14] discusses a method to measure the property degradation using asfabricated voxel model. The 3D object is built relying on deposition path after a reconstruction starting from an STL file, taking into account the importance of used resolution to achieve an higher order of detail in the inner structure made of voids and gaps. An experimental approach is used to determine this degradation. Three test specimens for tensile tests are manufactured at different building angle to detect its influence. Due to their complexity, lattice structures have to be replaced by a bulk material with equivalent mechanical properties in case of structure simulation to avoid computational memory saturation [57]. This process is called homogenization and it is done before FEA to decrease the computational time and costs. Reference [15] improves the discrete homogenization theory for lattices made by [52] considering semi-rigid joint effects. Joints are usually modelled as point of infinitesimal volume with infinite stiffness, without considering the truss decreasing length. For this new model, the concept of fixity (adjust joint stiffness) and eccentricity (adjust joint size with respect to truss diameter) of a joint is introduced. The basic concept behind the model is that each lattice beam is a series connection of 3 trusses (2 joints and a bar). The resulting semi-rigid joint stiffness matrix is a function of the material properties, geometries and joint parameters.

Increasing the component complexity in AM could lead to errors in the manufactured part, especially for lattice structures where the boundary surface is huge. There are a lot of issues that may affect the final product after additive processes, such as building direction and location, layer thickness, support structure. To quantify this problem, [24] introduces an hybrid approach that combines the B-rep visualization and voxel-based modelling. The authors develop a verification method between a virtual model from measurements, and the actual model: this is done using volumetric measurements (i.e. Computation Tomography (CT)).

It is worth discussing with the AM community the concept that there are numerous limitations related to the STL format, and times are mature to find a new format, more rich than the previous one, but fully compatible. As AM technology moves forward evolving towards multiple materials, like lattice structures, new formats are needed. Reference [31] tries to fill the gap for fiber-reinforced composites made by AM. The main idea is that all the areas with constant structure and variable size are modelled automatically and the file format is specialized for internal multi-material micro-structure. The new proposed file format is made of 3 sections: the first one contains parameters, representative volume elements and pores; the second one information about surface as STL format; while the last one the coefficient of polynomials describing the reinforced fibers in the domain, where maximum stress is expected.

Along this section, the main AM application have been discussed comprehensively. As a general comment, all the 25 contributions cover a wide range of tasks and 


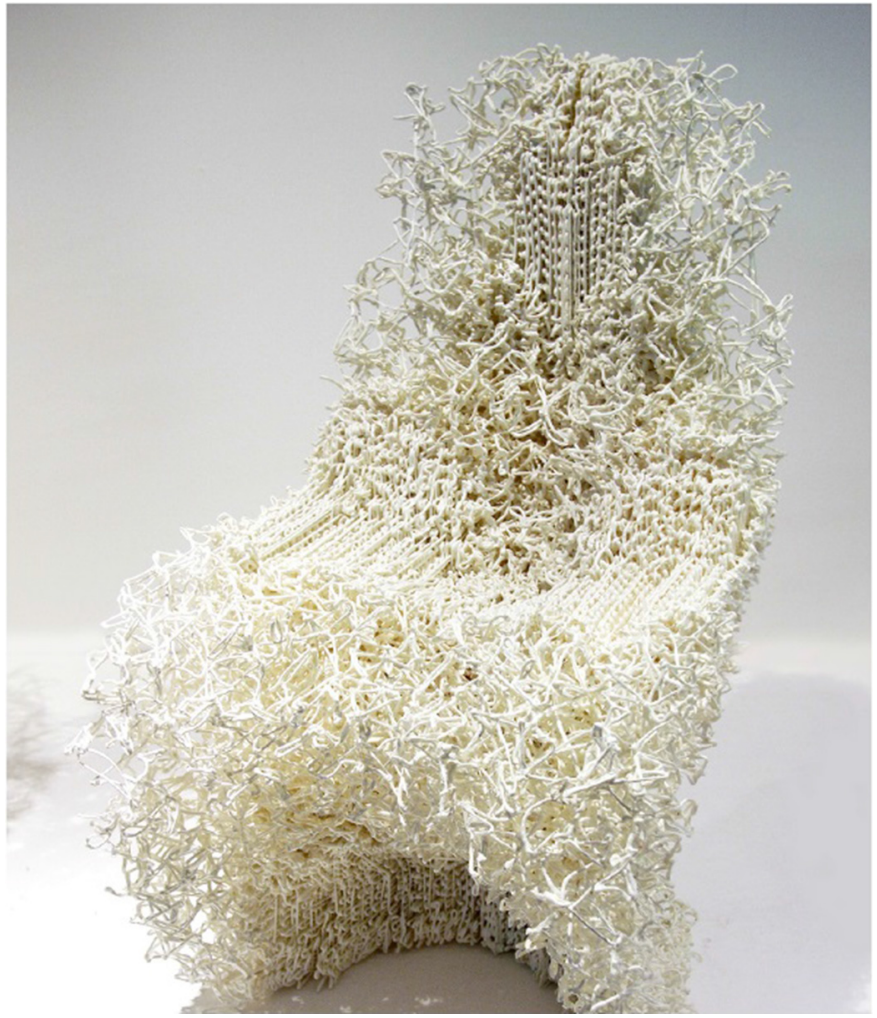

Fig. 13. Panton chair produced using robotic plastic deposition system [19].

applications that well reflects on the AM potentials and characteristics, but some limitations and possible areas of development are present, as it will be discussed in the following.

\subsubsection{AM technology}

The third characteristic here analysed, is the type of AM technology used in each bibliographic contribution. The overall statistics are collected in Figure 5. As already stated in this research, several AM techniques are mentioned by the authors in the 25 selected contributions.

The most recurring one $(38 \%)$ is the Fused Deposition Modelling (FDM) due to its low costs and wire material availability. In $[11,20]$ this technology is used as a basis for the simulation process framework, due to wide number of users and its enormous number of applications. In [28], where a Virtual Manufacturing simulator is developed, FDM technology is used because it holds the largest market share and many research results are available in literature. Deposition of fused plastic is also used in large $3 \mathrm{D}$ objects as in [19] to produce a Panton chair with complex inner geometry (Fig. 13). Besides, [14-16] use FDM to manufacture optimized specimens that are used in the experimental tests to validate the proposed models and methodologies. ABS plastic is cheap, easy to be found and is modelled by FDM machines, which are very wide spread in university and practitioners and a large community lie behind this technology. For very similar reasons, also in [32] authors exploit FDM technology to validate

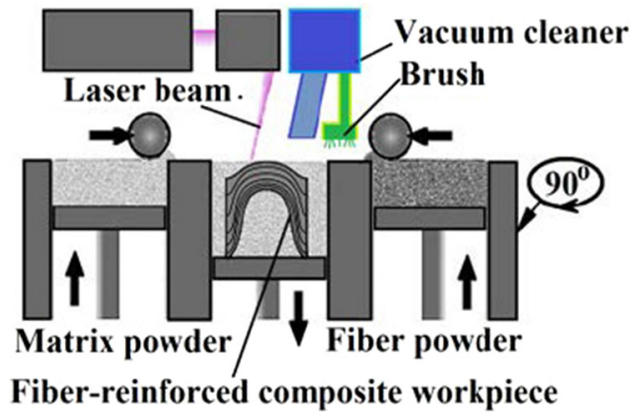

Fig. 14. Additive manufacturing machine for composite parts [31].

the proposed method. Even if advantages of this technology are clear, it is important to underline some limitations as well as: layer resolution, stair effect on the surface, component anisotropy, low strength and so on. This is the reason why, the proposed method in [24] to evaluate the "distance" between the manufactured part in FDM and the 3D model is an interesting contribution. Lastly, [9] proposes an optimized infill methodology for biomedical employment; the authors demonstrate the algorithm results using both FDM and SLS techniques.

Moving towards SLS and SLM, they are the basic technology considered by [21], where a framework for manufacturing quoting for SLS process is developed, due to its wide application in high-level engineering application where metallic parts are obtained. The tool allows forecasts on building time, material waste, energy consumption estimation, in almost real-time. Reference [31] develops a new file format for fiber-reinforced composite parts, where the manufacturing machine is based on the SLS concept. As a matter of fact, matrix powder is to be placed in one direction and fiber powder system placed in a perpendicular way (see machine layout in Fig. 14).

Reference [30] proposes an algorithm to produce stochastic and aperiodic procedural Voronoi microstructures, which sends to an AM machine either sliced images (SLA) or extract contours (SLS) to show and validate the proposed methodology.

Another recurring technology cited in the contributions is the stereolithography (SLA). Reference [13] develops a framework for material hollowing in order to minimize material consumption. Using SLA technique, material support is needed to assist the component growth layer by layer. Due to hollowing, external and internal material support is needed; this is optimized and minimized using a k-means clustering, taking into account the material strength. SLA is also used in [22] to build a prototype of multi-material FGM while in [25] it is used to manufacture some specimens for micro additive characterization. Different specimens at different iteration are obtained in order to investigate minimum printable feature, minimum feature distance and minimum layer thickness for the technology in question.

Material jetting, based on polyjet or inkjet technique, is mentioned in [26] because the best technology for the project purpose has been selected: each slice is described as a bitmap using the half-toning technique. This is done 
in order to design simultaneously geometry and material for graded components in AM, applied in prosthetics world, where high customization is needed. Material jetting is also used in [12] to validate the proposed model. This manufacturing technique is chosen because of the lack of material support for short-fiber micro-structure, at the basis of the discussed methodology.

Stand-alone example of application of LENS (Laser Engineered Net Shaping) technique can be seen in [10]. The thermal process simulation modelled in this paper takes into account a laser source that induces melting and bonding of the powder stock material.

Other examples of engineering applications outside the high-level industrial sector are described in $[33,27]$. The first one discusses the characterization of an assembler system for glass fiber and resin macro lattice unit cells. The second one develops a model for functionally graded building restoration using lightweight concretebased materials.

From this analysis regarding the materials and techniques involved in the selected contributions, it emerges that the designer has a wide choice of materials, but depending on application, economical budget and project constraints, there is always a preferred technology with respect to the others, that can be suggested by a careful reading of a milestone reference such as [1].

\subsubsection{Software platform}

Regarding the software platform used by the authors to develop their works, for some contributions the information is incomplete. Nevertheless, using the SLR methodology, authors are able to argue that the more common languages and software platforms are Matlab [58], OpenCL [59] and the couple Rhino \& Grassoppher.

In particular, Matlab, thanks to its enormous number of add-ons, libraries and routines, is used as software platform and as mathematical problem solver in $[16,20,21]$. Besides, since the scope of this work is to discuss the state of the art of voxelization too, authors would like to emphasize that, in addition to software platform, Matlab is used for component voxelization in $[11,13,17]$ because of its calculus speed capability, easy debugging, easiness in programming and user-friendly interface.

OpenCL is a standard for cross-platform, parallel programming of multi-processor boards found in personal computers, servers, mobile devices and embedded platforms (source: https://www.khronos.org/opencl/). In combination with $\mathrm{C}$ programming language, this programming standard is used as software platform for the $3 \mathrm{D}$ virtual manufacturing simulator described in $[28,29]$. Moreover, [30] uses OpenCL for procedural foam implementation as a kernel to process each foam slice.

Finally, the last software platform under analysis is Grasshopper (GH), a graphical algorithm editor tightly integrated with Rhino's 3D modelling tools (source: https://www.grasshopper3d.com/). GH is used in [23] to develop an add-on in order to implement the proposed simulation scheme A similar approach has been followed in [27], where $\mathrm{GH}$ is also used to design in a parametric

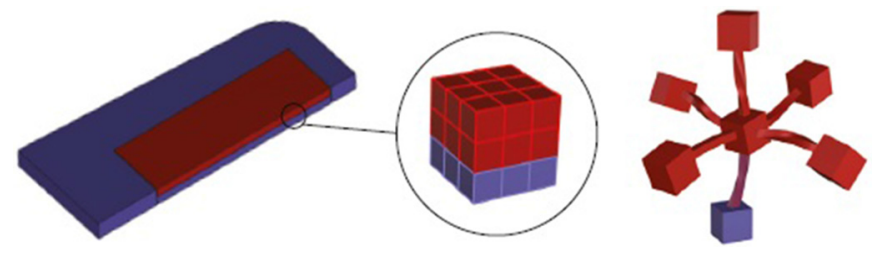

Fig. 15. Voxel application as matter constituent in a massspring system [23].

way 3D objects. [19] exploits the GH capability to translate the discretized and optimized toolpath for the robot plastic extrusion system.

\subsubsection{Voxelization usage}

As previously stated, voxelization is a discretization method based on the exploitation of small elements, mainly cubes, to approximate an external surface, or a 3D model, in order to speed up geometrical and algebraic manipulation (i.e. rotation, boolean operation, translation and so on) [60]. Like all the approximation methods, the smaller the elements, the more precise is the resulting representation: obviously this is true provided that all the available computational power is not saturated. The advantages of this method are the strength (voxelization rarely fails, unlike classic meshing), low memory consumption (all the element stiffness matrices are identical) and complex geometry can be handled easily.

As Figure 6 states, in the SLR methodology applied in this paper, voxels are mainly used as geometrical discretization or as material deposition unit. Only two stand-alone contributions assign to the voxel other tasks.

Particularly interesting is the application described in [23], where the authors used voxels as matter constituent, substituting points in the mass-spring system with the cuboid elements to ease the cognitive aspect of the particular design activity (Fig. 15).

Another stand-alone voxel application is described in [32]. Here voxels are used in a bi-dimensional environment. In this case, voxel is substituted by the analogue 2D element, the well known pixel. This is used as unitary element of CT images employed for inner structure reconstruction of bones that have to be additively manufactured.

A significant amount of the resulting contributions of the SLR methodology uses voxels as material deposition unit. After a deeper analysis, it emerges that [12] develops a methodology that is able to drive a voxel-based fabrication using material jetting. Thanks to this technique, it is possible to place material at the voxel level $(\mu \mathrm{m})$ to create complex shapes. Reference [31] relates each voxel to a powder particle or laser beam diameter of the described AM process: the developed file format drives the laser source in order to solidify the material voxel by voxel for better detail manufacturing. Reference [16] uses voxels to simulate the filament deposition process in a $3 \mathrm{D}$ voxelized volume, by activating in a binary way the corresponding element where new material is added (i.e. transforming the voxel value from 0 to 1 ). A completely 

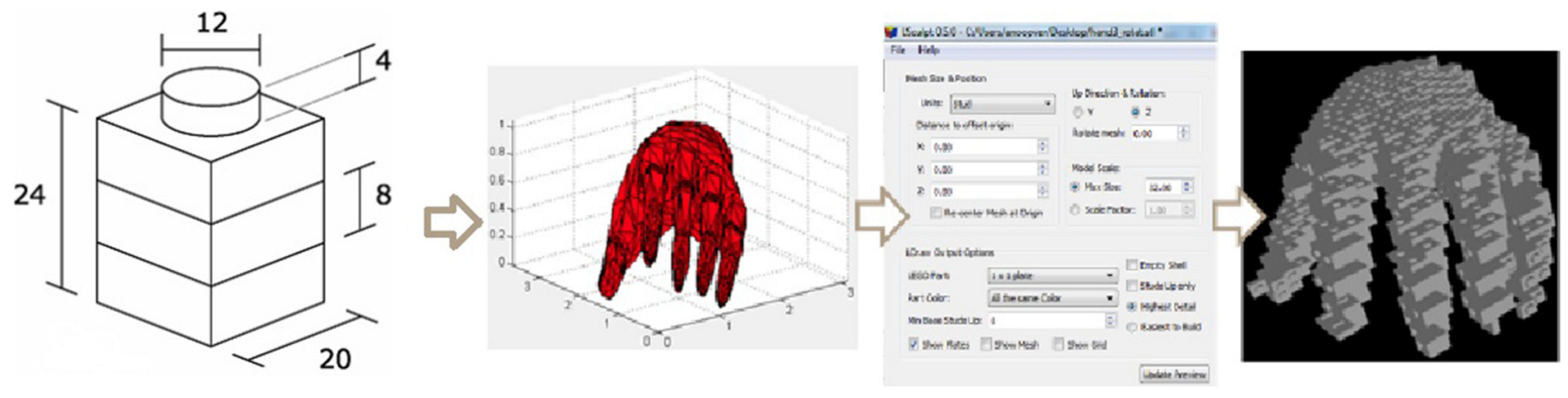

Fig. 16. Lego studs elements for geometry discretization [21].

different application is described in [33], where macro voxels are mentioned, representing the deposition of a macro unit cell of lattice structure, assimilating to this category of voxel employment. On the other hand, regarding microadditive manufacturing, [25] uses cuboid elements whose dimensions $(\mu \mathrm{m})$ represent the smallest feature achievable with the described AM technology. Another contribution that belongs to this category, where voxels are used as material deposition unit, is identified in [22]. Here the authors use cuboid elements as minimal volume of simple material that can be placed to manufacture an object but also as computational unit: a material property between two available boundary materials is associated based on a gradient function, in order to generate a functionally graded material. A further hybrid employment of voxel as material deposition unit, because of material jetting choice and geometry discretization unit, is shown in [26] to speed up manipulation operations for CT image comparison.

As the statistics show, analysing all the 25 contributions, $64 \%$ of them use voxels as a geometrical discretization unit and as support to computational operations. This is because voxelization is an efficient way to store geometrical information in a discrete way, transforming a 3D model into an integer 3D data matrix made of 0 and 1 . As done before, each paper has been carefully analysed to get important information about the voxel use state of the art.

All the analysed contributions use standard cuboid elements with the exception of [21] where authors use lego studs to handle complex geometries in an easier way (Fig. 16).

Reference [9] uses voxels as computational unit for topology optimization evaluation, in a similar manner respect to what done in $[10,29]$, where the discretized elements are used as a support for the thermal process simulation. There are some papers associated with the same voxel use: $[11,15,28]$ who use these elementary cubes as a computational unit to discrete the geometry under analysis in order to detect critical areas (i.e. under or over-extrusion regions and generic geometrical errors) and evaluate the support material location by minimizing it, as done in [13]. Here, support material is needed to manufacturing the part because of voxel binary hollowing computation to optimize the component.

There are also some contribution where a particular voxel employment, always as geometry discretization unit, has been identified. In particular, $[18,27]$ develop a
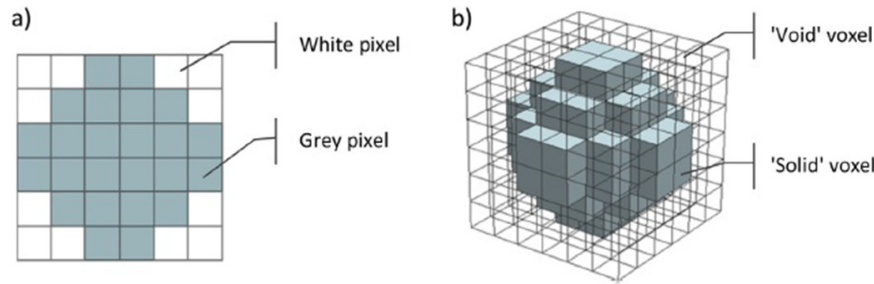

Fig. 17. Parallelism of $2 \mathrm{D}$ pixels and $3 \mathrm{D}$ voxels using black and white geometry description [17].

voxel-based finite element analysis to evaluate stress distribution by using a black and white mesh. The same binary approach, using a volumetric representation based on elementary cubics of 1 and 0, is employed in [24], where authors try to enrich a well known voxelization representation with product and manufacturing information (as layer, material and mating surface ID), prior to writing the geometry description file.

An interesting application of voxels for material optimization and lightweight structure design is described in [17]. The authors define the volume of a certain shape in a bitwise way in order to speed up geometrical operations such as surface offsetting by considering only boundary elements (Fig. 17). The Ray Tracing voxelization method, inspired from [61], is used as a basis for lattice generation by volume tessellation, substituting boundary or functional representations not optimized for this kind of structure.

Other important contributions to the field are given by $[14,19]$. The first one uses geometrical voxelization to access part degradation, based on the AM tool deposition path, while the second one uses cuboid elements as a computational unit to optimize and discretize the machine toolpath combining neighbour voxels depending on the stress amount detected using FEM analysis. And last but not least contribution [20] uses voxels to simultaneously compute design and processing factors to evaluate material support using a black and white geometry definition to speed up the simulation.

As the reader can understand, voxelization is very common in the Additive Manufacturing field where prior to produce parts, computer software have to handle complex shapes. This discretization technique, used for different purposes, enables strong, efficient and fast volume rendering without obstructing the model fidelity, even if complex 


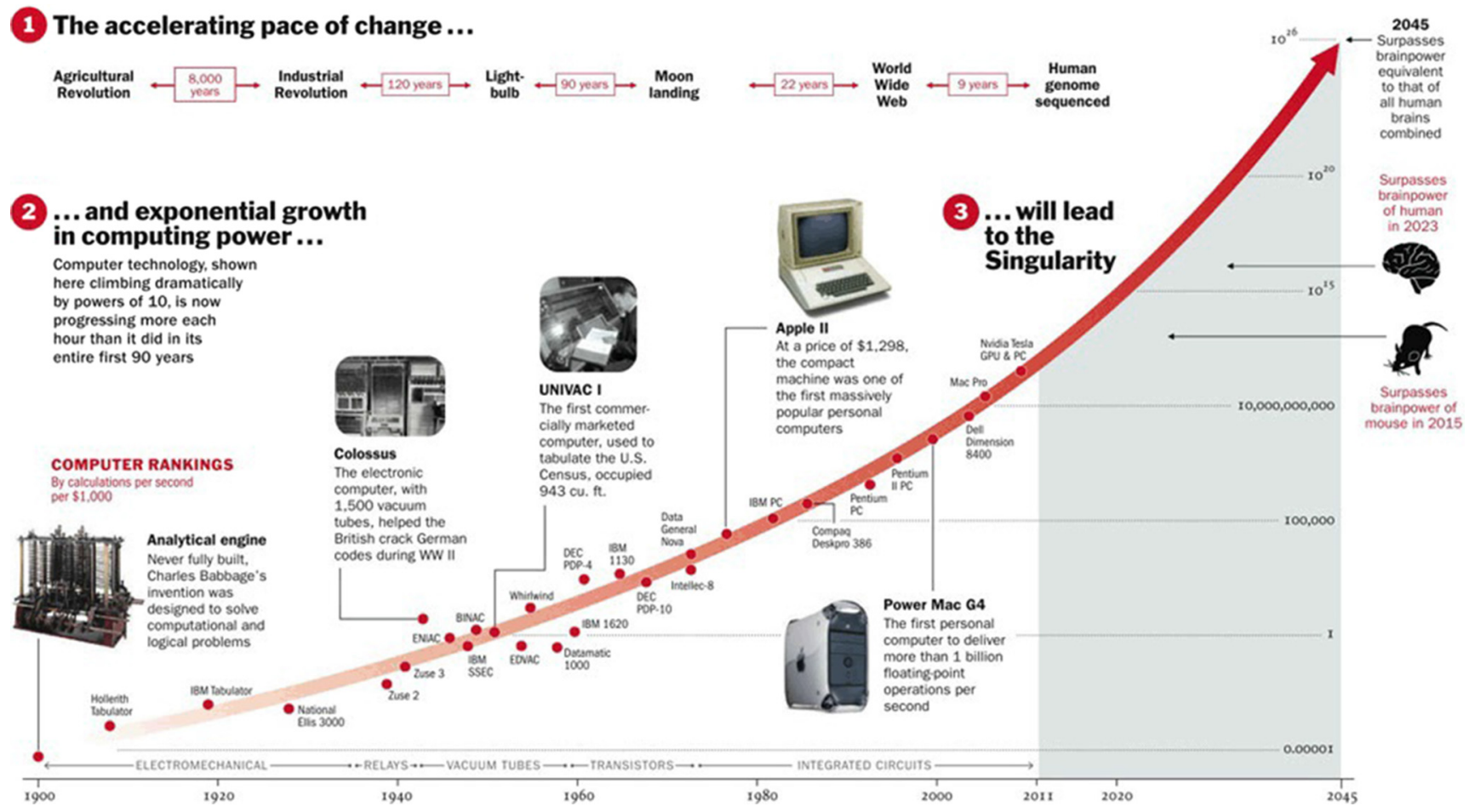

Fig. 18. Computational power growth during the last century (Source: Time Magazine).

geometrical operation have to be done before manufacturing process starts, demonstrating great potentiality for future developments.

\subsection{Answer to Q2: What are the potential future developments and possible new implementation of voxel-based algorithms in additive manufacturing?}

In order to answer the second research question, the 25 contributions are analysed in the discussion, future work and conclusion sections. This is done to depict the future developments of the technology the paper is about. In particular, there is a general trend to assume that more efficient and accurate results, whatever they are, can be possible by the increasing computational power. As [18] explains, it is interesting to see how the voxel mesh size affects the results: a finer mesh reflects on more robust and finer results compared to a coarse mesh. By the way, technology is rapidly evolving and the computational power available is increasing year by year as (Fig. 18) shows.

For the aim of this paper, it is clear that with a bigger computational resource a finer discretized voxel-based model can be achieved. For example, [17] explains the interaction of computational power and proposed lattice design methodology: an increase of voxels describing the geometry translates into the increase of the model accuracy, especially for external smooth surfaces. Reference [24] states that with more computational power, the proposed voxel-based representation can be extended also for assemblies, enriching the proposed voxel file format with part identification number. As a matter of fact, an increase of computational power reflects on the presented methodologies of this SLR by an increasing of the result accuracy, reduction of computational time and time needed for the design-to-manufacturing cycle.

Analysing each contribution, [10] would like to extend the thermal simulation also for phase-change flows and not only for the transient ones, making the simulation framework more complete. Reference [12] proposes to extend the topology optimization model for multi-physics simulations, increasing the already good framework capabilities. A similar proposal is described in [14], where an integration of thermal influence during manufacturing process has been associated to an increase in the lattice degradation analysis results.

In $[11,13,21]$ authors in the future would like to extend the quoting capability by experimental validation, modifying the fitness functions in case of maximization or minimization of a certain physical quantity by incorporating other interconnected parameters to get an overall accuracy increase. The same target is described in [25], where authors would like to refine the AM machine characterization in order to get more accurate models in terms of minimum printable features. Reference [30] explains how interesting it would be to extend the proposed stochastic design methodology to the anisotropic foams and implementing a variable Poisson ratio value, as it has been done with the Young modulus, while [22] would like to extend the methodology from $2.5 \mathrm{D}$ to $3 \mathrm{D}$ components. Some analysed papers $[20,27,28]$ propose their relative models and methodologies in an analytical way and intend to validate them by an experimental approach.

A relevant group of papers focuses on the lattice structures, that could bring important weight and fuel consumption reduction in the transportation field. In particular, [15] proposes to refine the model with end effects 
for non periodic lattices and try to model analytically and experimentally the buckling behaviour of these complex structures. On the other hand, for biomedical purposes, [32] has proposed to extend the inner structure reconstruction by means of $\mathrm{CT}$ images not only to uniform lattices, but also to the graded ones, based on grey scale image superposition.

A group of papers highlights the CAD limitations for AM application and tries to propose alternative representation methodologies for future developments. In particular, [19] has tried to fill the gap between computation and fabrication by substituting the $3 \mathrm{D}$ printer concept with a voxel printer that is able to allocate a discrete amount of material in a given position. Reference [26] has proposed to increase the level of control of printing process to create optimized functionally multi-materials by changing the way a $3 \mathrm{D}$ model is described, i.e the file format. As states [1], it is well known that the STL file format shows limitations in terms of material description for lattice structures, multi-materials and texture surfaces. The ASTM Committee releases a new standard for the Additive Manufacturing File Format (AMFF) that is under development. With respect to the STL one, this new kind of file can integrate information about curved triangles, colour, texture, material and its variants.

From the SLR point of view, it was identified that several interesting project are available in literature, even if some limitation are present. Different frameworks and developed mathematical models in the AM field using voxel-based representation constitute the background for further improvements. Despite this, it seems that each contribution can be integrated with the others in a better way, as proposed in [16], by using as platform an opensource CAD tool with different environments, where each task corresponding to the different developed frameworks could be integrated (i.e. manufacturing quoting, structural analysis, topology optimization, thermal analysis and so on). A possible solution could be FreeCad, a free and opensource CAD software in which different macros and workbenches, coded in Python language can be integrated and shared between the huge community (See as example [62]).

Another important step for the engineering and computer science community can be the voxel employment as a discretization unit for fluid-structural interaction (FSI) simulations, very important in the automotive sector, especially in racing cars and motorbikes. Such discretization method can be used as a support to solve the numerical problem and voxel-based discretization units can represent complex shapes ready to be manufactured using AM technology.

\section{Conclusions and future works}

The aim of this paper is to analyse the available literature contributions on voxel-based methods applied to Additive Manufacturing. This is carried out by answering two research questions: (Q1) What is the state of the art of voxel discretization algorithms to be used for typical complex shapes to be manufactured with
Additive Manufacturing technologies? (Q2) What are the potential future developments and possible new implementation of voxel-based algorithms in Additive Manufacturing?

A selective literature review methodology (SLR) is applied to filter the large amount of publications available in research data bases in order to get the most relevant ones. This approach is chosen in order to provide a fully reproducible methodology where a subjective point of view belongs only to the quality criteria assigned to each contribution. SLR was able to extract from the database 25 contributions, starting from 184 articles belonging to four database (duplicates included). The most relevant 25 papers have been deeply analysed to get information about: field of application, voxel use, additive manufacturing technology, software platform selected to develop algorithms among others.

The data extraction process has been carefully explained in the paper with the support of graphic flow charts. Results and statistics are collected and shown to the reader in pie charts for an immediate understanding of the analysed literature features.

From the relevant contributions, it emerges that the main challenges to apply voxel-based methods in AM relates the computational power available by hardware tools, with a finer discretization producing more accurate results. Nevertheless, voxelization process is based upon a strong discretization algorithm able to store geometrical information in discrete and efficient way that rarely fails. The voxel model description is used to simulate AM process using different manufacturing technologies, or to support demanding computations like that required by topology optimization, lattice modelling and analysis and so on.

Authors believe that the literature review included in this paper can support researchers and companies research and development $(\mathrm{R} \& \mathrm{D})$ departments, being a starting point to understand the state of the art, technology gaps and suggesting possible areas of development.

Future works could focus on the integration of different voxel-based application in AM as discussed in this paper. In particular, voxels could be used in fluid-structure interaction applications, for the discretization and geometry handling, and to mesh bodies in structural and fluid analysis.

\section{References}

[1] I. Gibson, D. Rosen, B. Stucker, Additive manufacturing technologies, 2015.

[2] W. Gao, Y. Zhang, D. Ramanujan, K. Ramani, Y. Chen, C.B. Williams, C.C. Wang, Y.C. Shin, S. Zhang, P.D. Zavattieri, The status, challenges, and future of additive manufacturing in engineering, Comp. Aided Des. 69, 65-89 (2015)

[3] G. Jense, Voxel-based methods for CAD, Comp. Aided Des. 21, 528-533 (1989)

[4] A.E. Kaufman, Volume visualization, ACM Comput. Surv. 28, 165-167 (1996)

[5] D.P.A. Booth, A. Sutton, Systematic approaches to a successful literature review, 2011 
[6] B. Kitchenham, O.P. Brereton, D. Budgen, M. Turner, J. Bailey, S. Linkman, Systematic literature reviews in software engineering - a systematic literature review, Inf. Softw. Technol. 51, 7-15 (2009)

[7] P. Brereton, B.A. Kitchenham, D. Budgen, M. Turner, M. Khalil, Lessons from applying the systematic literature review process within the software engineering domain, J. Syst. Softw. 80, 571-583 (2007)

[8] A.C.C. dos Santos, M.E. Delamaro, F.L. Nunes, The relationship between requirements engineering and virtual reality systems: a systematic literature review, in 2013 XV Symposium on Virtual and Augmented Reality, IEEE, 2013

[9] J. Wu, N. Aage, R. Westermann, O. Sigmund, Infill optimization for additive manufacturing|approaching bone-like porous structures, IEEE Trans. Visual. Comput. Graph. 24, 1127-1140 (2018)

[10] A. Krishnakumar, K. Suresh, A. Chandrasekar, Towards assembly-free methods for additive manufacturing simulation, in 35th Computers and Information in Engineering Conference, ASME, Volume 1A, 2015

[11] S.E. Ghiasian, P. Jaiswal, R. Rai, K. Lewis, From conventional to additive manufacturing: Determining component fabrication feasibility, Vol. 2A-2018, Quebec City, QC, Canada, 2018

[12] N. Boddeti, Z. Ding, S. Kaijima, K. Maute, M.L. Dunn, Simultaneous digital design and additive manufacture of structures and materials, Sci. Rep. 8, 15560 (2018)

[13] U.S. Venkatesan, S. Pande, Efficient process planning strategies for additive manufacturing, Vol. 2, Los Angeles, CA, United states, 2017

[14] S. Park, D.W. Rosen, Quantifying effects of material extrusion additive manufacturing process on mechanical properties of lattice structures using as-fabricated voxel modeling, Addit. Manufactur. 12, 265-273 (2016)

[15] S.-I. Park, D.W. Rosen, Homogenization of mechanical properties for material extrusion periodic lattice structures considering joint stiffening effects, J. Mech. Des. 140, 111414 (2018)

[16] A. Gleadall, I. Ashcroft, J. Segal, VOLCO: a predictive model for 3d printed microarchitecture, Addit. Manufact. 21, 605-618 (2018)

[17] A. Aremu, J. Brennan-Craddock, A. Panesar, I. Ashcroft, R. Hague, R. Wildman, C. Tuck, A voxel-based method of constructing and skinning conformal and functionally graded lattice structures suitable for additive manufacturing, Addit. Manufact. 13, 1-13 (2017)

[18] G.A. da Silva, A.T. Beck, O. Sigmund, Stress-constrained topology optimization considering uniform manufacturing uncertainties, Comput. Methods Appl. Mech. Eng. 344, 512-537 (2019)

[19] G. Retsin, M.J. Garcia, Discrete computational methods for robotic additive manufacturing, ACADIA, 2016

[20] S. Dinda, D. Modi, T.W. Simpson, S. Tedia, C.B. Williams, Expediting build time, material, and cost estimation for material extrusion processes to enable mobile applications, in Volume 2A: 43rd Design Automation Conference, ASME, 2017.

[21] A. Verma, R. Rai, Computational geometric solutions for efficient additive manufacturing process planning, Vol. 1A, Buffalo, NY, United states, 2014

[22] Y.-S. Leung, H. Mao, Y. Chen, Approximate functionally graded materials for multi-material additive manufacturing, Vol. 1A-2018, Quebec City, QC, Canada, 2018
[23] G. Sossou, F. Demoly, G. Montavon, S. Gomes, Design for $4 \mathrm{~d}$ printing: rapidly exploring the design space around smart materials, Proc. CIRP 70, 120-125 (2018)

[24] G. Moroni, S. Petrò, W. Polini, Geometrical product specification and verification in additive manufacturing, CIRP Ann. 66, 157-160 (2017)

[25] M.K. Thompson, M. Mischkot, Design of test parts to characterize micro additive manufacturing processes, Proc. CIRP 34, 223-228 (2015)

[26] E. Doubrovski, E. Tsai, D. Dikovsky, J. Geraedts, H. Herr, N. Oxman, Voxel-based fabrication through material property mapping: a design method for bitmap printing, Comp. Aided Des. 60, 3-13 (2015)

[27] F. Craveiro, H. Bartolo, A. Gale, J. Duarte, P. Bartolo, A design tool for resource-efficient fabrication of 3d-graded structural building components using additive manufacturing, Autom. Constr. 82, 75-83 (2017)

[28] S.-K. Ueng, L.-G. Chen, S.-Y. Jen, Voxel-based virtual manufacturing simulation for three-dimensional printing, Adv. Mech. Eng. 10, 168781401878163 (2018)

[29] A. Ripetskiy, S. Zelenov, E. Kuznetsova, L. Rabinskiy, Evaluation of the thermal processes and simulation methods for additive manufacturing based on the geometry voxel representation, Key Eng. Mater. 771, 91-96 (2018)

[30] J. Martínez, J. Dumas, S. Lefebvre, Procedural voronoi foams for additive manufacturing, ACM Trans. Graph. 35, 1-12 (2016)

[31] E. Shchurova, A. Shchurova, A new file format to describe fiber-reinforced composite workpiece structure for additive technology machines, Proc. Eng. 129, 105-110 (2015)

[32] A. Ahsan, R. Xie, B. Khoda, Heterogeneous topology design and voxel-based bio-printing, Rapid Prototyp. J. 24, 11421154 (2018)

[33] O. Formoso, G. Trinh, S. Hu, K. Cheung, Development and robustness characterization of a digital material assembly system, Proc. Manufact. 26, 1003-1013 (2018)

[34] R. Palmarini, J.A. Erkoyuncu, R. Roy, H. Torabmostaedi, A systematic review of augmented reality applications in maintenance, Robot. Comput. Integr. Manufact. 49, 215228 (2018)

[35] M.P. Bendsoe, O. Sigmund, Topology optimization - Theory, methods and applications, 2004.

[36] ASTM standard f2792, standard terminology for additive manufacturing technologies, Tech. rep., ASTM International, 2013

[37] D. Pham, R. Gault, A comparison of rapid prototyping technologies, Int. J. Mach. Tools Manuf. 38, 1257-1287 (1998)

[38] M.K. Agarwala, V.R. Jamalabad, N.A. Langrana, A. Safari, P.J. Whalen, S.C. Danforth, Structural quality of parts processed by fused deposition, Rapid Prototyp. J. 2, 4-19 (1996)

[39] P.M. Pandey, N.V. Reddy, S.G. Dhande, Slicing procedures in layered manufacturing: a review, Rapid Prototyp. J. 9, 274-288 (2003)

[40] H. Le, Progress and trends in ink-jet printing technology, J. Imag. Sci. Technol. 42, 49-62 (1998)

[41] B.-J. de Gans, P.-C. Duineveld, U.-S. Schubert, Inkjet printing of polymers: State of the art and future developments, Adv. Mater. 16, 203-213 (2004)

[42] J.J. Beaman, C.R. Deckard, Selective laser sintering with assisted powder handling

[43] E. Olakanmi, R. Cochrane, K. Dalgarno, A review on selective laser sintering/melting (SLS/SLM) of aluminium alloy 
powders: Processing, microstructure, and properties, Progr. Mater. Sci. 74, 401-477 (2015)

[44] M.L. Griffith, L.D. Harwell, T. Romero, E. Schlienger, C.L. Atwood, J.E. Smugeresky, Multi-material processing by lens, in: University of Texas at 1997, pp. 387-393

[45] K. Cooper, Rapid Prototyping Technology, CRC Press, 2001

[46] G. Trinh, G. Copplestone, M. O'Connor, S. Hu, S. Nowak, K. Cheung, B. Jenett, D. Cellucci, Robotically assembled aerospace structures: digital material assembly using a gantry-type assembler, in 2017 IEEE Aerospace Conference, IEEE, 2017

[47] B. Khoshnevis, D. Hwang, K.T. Yao, Z. Yeh, Mega-scale fabrication by contour crafting, Int. J. Ind. Syst. Eng. 1, 301 (2006)

[48] T.D. Ngo, A. Kashani, G. Imbalzano, K.T. Nguyen, D. Hui, Additive manufacturing ( $3 \mathrm{~d}$ printing): a review of materials, methods, applications and challenges, Compos. Part B 143, 172-196 (2018)

[49] S. Singh, S. Ramakrishna, R. Singh, Material issues in additive manufacturing: a review, J. Manufact. Process. 25, 185-200 (2017)

[50] O. Sigmund, A 99 line topology optimization code written in matlab, Struct. Multidiscipl. Optim. 21, 120-127 (2001)

[51] D.S. Nguyen, F. Vignat, A method to generate lattice structure for additive manufacturing, in: 2016 IEEE International Conference on Industrial Engineering and Engineering Management (IEEM), IEEE, 2016

[52] A. Vigliotti, D. Pasini, Stiffness and strength of tridimensional periodic lattices, Comp. Methods Appl. Mech. Eng. 229-232, 27-43 (2012)
[53] K. Sai Nithin Reddy, V. Maranan, T.W. Simpson, T. Palmer, C.J. Dickman, Application of topology optimization and design for additive manufacturing guidelines on an automotive component, in: Volume 2A: 42nd Design Automation Conference, ASME, 2016

[54] M. Zhou, G. Rozvany, The COC algorithm, part II: topological, geometrical and generalized shape optimization, Comp. Methods Appl. Mech. Eng. 89, 309-336 (1991)

[55] N.P. Suh, The principles of design, Oxford University Press Inc, 1990

[56] M. McMillan, M. Jurg, M. Leary, M. Brandt, Programmatic lattice generation for additive manufacture, Proc. Technol. 20, 178-184 (2015)

[57] S. Arabnejad, D. Pasini, Mechanical properties of lattice materials via asymptotic homogenization and comparison with alternative homogenization methods, Int. J. Mech. Sci. 77, 249-262 (2013)

[58] MathWorks, Matlab, www.mathworks.com/products/ matlab.html

[59] K. Group, Opencl, www.khronos.org/opencl

[60] D. Cohen-Or, A. Kaufman, Fundamentals of surface voxelization, Graph. Models Image Process. 57, 453-461 (1995)

[61] E.-A. Karabassi, G. Papaioannou, T. Theoharis, A fast depth-buffer-based voxelization algorithm, J. Graph. Tools 4, 5-10 (1999)

[62] A. Ceruti, R. Ferrari, A. Liverani, Design for additive manufacturing using LSWM: a cad tool for the modelling of lightweight and lattice structures, in G. Campana, R.J. Howlett, R. Setchi, B. Cimatti (Eds.), Sustainable Design and Manufacturing 2017, Springer International Publishing, Cham, 2017, pp. 756-765.

Cite this article as: A. Bacciaglia, A. Ceruti, A. Liverani, A systematic review of voxelization method in additive manufacturing, Mechanics \& Industry 20, 630 (2019) 